\title{
Outage Probability Analysis in Power-Beacon Assisted Energy Harvesting Cognitive Relay Wireless Networks
}

\author{
Ngoc Phuc Le \\ Institute of Research and Development, Duy Tan University, Da Nang, Vietnam \\ Correspondence should be addressed to Ngoc Phuc Le; phucln81@yahoo.com
}

Received 7 July 2017; Accepted 8 August 2017; Published 24 September 2017

Academic Editor: Nathalie Mitton

Copyright (C) 2017 Ngoc Phuc Le. This is an open access article distributed under the Creative Commons Attribution License, which permits unrestricted use, distribution, and reproduction in any medium, provided the original work is properly cited.

\begin{abstract}
We study the performance of the secondary relay system in a power-beacon (PB) assisted energy harvesting cognitive relay wireless network. In our system model, a secondary source node and a relay node first harvest energy from distributed PBs. Then, the source node transmits its data to the destination node with the help of the relay node. Also, fading coefficients of the links from the PBs to the source node and relay node are assumed independent but not necessarily identically distributed (i.n.i.d) Nakagami- $m$ random variables. We derive exact expressions for the power outage probability and the channel outage probability. Based on that, we analyze the total outage probability of the secondary relay system. Asymptotic analysis is also performed, which provides insights into the system behavior. Moreover, we evaluate impacts of the primary network on the performance of the secondary network with respect to the tolerant interference threshold at the primary receiver as well as the interference introduced by the primary transmitter at the secondary source and relay nodes. Simulation results are provided to validate the analysis.
\end{abstract}

\section{Introduction}

Energy harvesting (EH) has recently emerged as a promising approach to enable self-sustaining operation of energyconstrained wireless systems [1]. In EH-based communication systems, wireless devices scavenge energy from ambient energy sources, such as solar, wind, geothermal, and vibration sources [2]. In addition to these alternative energy sources, radio frequency $(\mathrm{RF})$ signals have been considered as another promising energy source since it owns several favorable properties including availability and controllability. As RF signal is capable of carrying both information and energy at the same time, the authors in [3] devised receiver architectures for simultaneous wireless information and power transfer (SWIPT) systems. However, due to a huge gap regarding the operational sensitivity level between an information decoder and an energy harvester, SWIPT systems are only suitable for short-range transmission. To overcome this disadvantage, the authors in [4] proposed a novel wireless-powered system architecture in which power-beacons (PBs) are deployed to power wireless devices. Several PB-assisted energy harvesting systems have been then proposed and investigated in the literature; see, for example, [5-10].

Since EH-based point-to-point systems have been well investigated in the literature, many research works have recently focused on investigating energy harvesting in complicated system architectures, such as relay/cooperative systems, cognitive radios, and cognitive relaying networks. For relay systems, two EH relaying protocols, namely, time switching-based relaying (TSR) and power splitting-based relaying (PSR), are frequently used for energy harvesting and signal processing at a relay node [11]. In particular, in the TSR protocol, the relay node harvests energy from the received signal in a portion of time and spends the rest of the time period for information receiving and processing. Meanwhile, for the PSR protocol, the relay splits the received signal from the source into two streams, one of which is used for energy harvesting operation and the other for information decoding. Throughput analysis of amplifyand-forward (AF) and decode-and-forward (DF) relaying systems was performed in [11] and [9], [12], respectively. In addition, several aspects associated with EH relay systems 
have been examined, including EH-based relaying networks in the presence of interference [13], EH-based cooperative systems with spatially random relays [14], relay selection for trade-off between quality of information transfer and average transferred energy [15], and two-way EH-based relay systems [16].

With respect to cognitive radio (CR), it is well established that CR networks could offer a significant improvement in terms of spectrum utilization efficiency by allowing unlicensed secondary network (SN) users to share the same spectrum licensed to the primary network [17]. Among different cognitive paradigms, an underlay mode in which SN users transmit in the same band with the primary transmitters simultaneously subject to an interference constraint at the primary receiver is the simplest and is easy to implement in practice. Therefore, many studies have considered energy harvesting for underlay CR networks; see, for example, [1822]. In [18], the authors analyzed the outage probability and spatial throughput of the SN users. They also derived the optimal transmission power and density of SN nodes, which provides insights into optimal network design. In [19], a harvested energy-throughput trade-off optimization problem was formulated and a closed-form solution was obtained. Optimal resource allocation (i.e., time sharing and power) for EH-based CR networks was considered in [20, 21]. Also, an extension to joint time and power allocation for maximal sum-throughput in multiuser EH-based CR networks was studied in [22].

Recently, some research works have considered energy harvesting for cognitive relay networks (CRN). The motivation behind this approach is to exploit the advantages of cognitive radio and relay techniques [23]. In [24], the authors investigated $\mathrm{EH}$-based underlay CRN with DF relaying and the TSR protocol. In their system model, secondary source and relay nodes harvest energy from multiple primary users that are closely located. They performed exact analysis of the outage probability as well as asymptotic analysis when the number of primary nodes goes to infinity. An analysis of outage probability in EH-based CRN with the PSR protocol was investigated in [25]. CRN with the PSR protocol was further investigated in [26] in which outage performance, energyrate trade-off, and energy efficiency metrics were analyzed and optimized. In addition, analysis of the outage probability in multihop CRN and CRN with opportunistic relay selection was studied in [27] and [28], respectively. It is worth noting that these studies assume that the energy harvesting circuitry at receivers is always activated. Fading channel coefficients are assumed to follow independent and identical distribution (i.i.d). Moreover, instantaneous channel state information (CSI) of the links between SN nodes and a primary receiver (PR) is available. In many practical scenarios, it might be hard to access the instantaneous CSI since the collaboration between SN nodes and primary receiver is very limited.

Unlike the existing works, we consider in this study a distributed power-beacons (PBs) assisted energy harvesting cognitive relay wireless network. Some distinguished features of our system model are as follows: (i) simple PBs equipped with single-antenna are deployed to broadcast energy and they perform no additional information processing tasks; (ii) secondary source node and relay node need to harvest energy from PBs (and a primary transmitter if available) to sustain information transmission operations but they might be inactive if the harvested power is not sufficient, (iii) fading coefficients of the channels from PBs to secondary nodes follow i.n.i.d Nakagami- $m$ distributions; and (iv) instantaneous CSI of the links between SN nodes and PR might be available or not (i.e., only statistical CSI). Our aim is to comprehensively analyze the outage probability of the secondary relay system under a spectrum sharing context with energy harvesting capability. The main contributions of this work can be summarized as follows:

(i) Derivation of analytical expressions for the power outage probability (i.e., the probability that the harvested power at the source node and/or relay node is below the minimum circuit activation power threshold) and the channel outage probability (i.e., the probability that the signal-to-noise ratio (SNR) of the received signal at the destination node is below the SNR threshold).

(ii) Derivation of the total outage probability of the secondary network in both noise-limited scenario (i.e., the primary transmitter is far away from the secondary network) and interference-limited scenario (i.e., the primary transmitter is near to the secondary network).

(iii) Derivation of the total outage probability of the secondary network subject to the availability of the instantaneous CSI or statistical CSI of the links between the secondary network and the primary receiver.

(iv) Asymptotic analysis of the outage probability of the secondary relay system under different scenarios, which provides important insights into the behavior of the outage performance.

(v) Evaluation of impacts of the transmit power of powerbeacons, the tolerant interference threshold at the primary receiver, and the transmit power of the primary transmitter on the outage probability of the secondary relay system.

The rest of the paper is organized as follows. In Section 2, we describe the cognitive relay system model with RF energy harvesting. In Section 3, we analyze the power outage probability. Exact and asymptotic analysis of the total outage probability of the secondary relay system are carried out in Section 4. Simulation results and discussions are provided in Section 5. Finally, we conclude the paper in Section 6.

\section{System Model}

We consider a distributed PB-assisted energy harvesting cognitive relay wireless network as depicted in Figure 1. All nodes are equipped with single-antenna. The secondary network consists of a source node (SS), a decode-and-forward (DF) relay node (SR), and a destination node (SD). The source node and relay node are energy-constrained devices that need to harvest energy from $N$ PBs for data transmission. Also, the 
TABLE 1: Symbol notations.

\begin{tabular}{|c|c|c|c|c|}
\hline Links & Fading coefficient & Distance & Squared mean & Path-loss at $1 \mathrm{~m}$ \\
\hline$\overline{\mathrm{PB}_{i}-\mathrm{SS}}$ & $h_{i s}$ & $d_{i s}$ & $\Omega_{i s}$ & $L_{i s}^{0}$ \\
\hline $\mathrm{PB}_{i}-\mathrm{SR}$ & $h_{i r}$ & $d_{i r}$ & $\Omega_{i r}$ & $L_{i r}^{0}$ \\
\hline SS-SR & $h_{s r}$ & $d_{s r}$ & $\Omega_{s r}$ & $L_{s r}^{0}$ \\
\hline SR-SD & $h_{r d}$ & $d_{r d}$ & $\Omega_{r d}$ & $L_{r d}^{0 t}$ \\
\hline SS-PR & $h_{s p}$ & $d_{s p}$ & $\Omega_{s p}$ & $L_{s p}^{0}$ \\
\hline SR-PR & $h_{r p}$ & $d_{r p}$ & $\Omega_{r p}$ & $L_{r p}^{0 P}$ \\
\hline PT-SS & $h_{t s}$ & $d_{t s}$ & $\Omega_{t s}$ & $\begin{array}{l}L_{p}^{p} \\
L_{t s}^{0}\end{array}$ \\
\hline PT-SR & $h_{t r}$ & $d_{t r}$ & $\Omega_{t r}$ & $L_{t r}^{0}$ \\
\hline PT-SD & $h_{t d}$ & $d_{t d}$ & $\Omega_{t d}$ & $L_{t d}^{0}$ \\
\hline
\end{tabular}

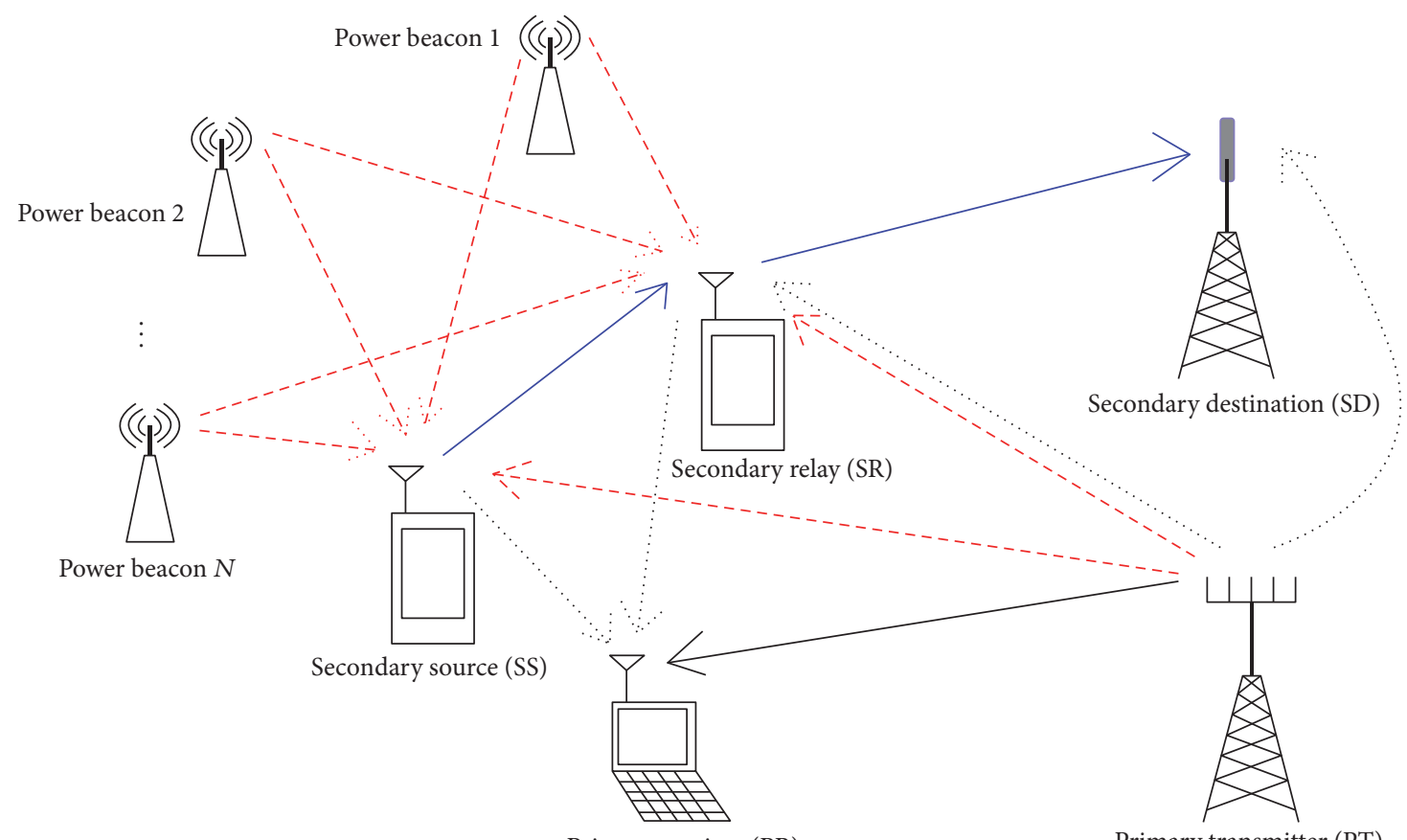

Primary receiver $(\mathrm{PR})$

Primary transmitter (PT)

\footnotetext{
- - Power transfer

_ Information transmission

...... Interference
}

Figure 1: System model.

direct link between SS and SD is assumed not to exist due to poor fading conditions or obstacles. Hence, data transmission from SS to SD is accomplished with the help of SR. In this work, we consider an underlay cognitive paradigm, in which the secondary nodes can perform data transmission in the same spectrum band licensed to the primary network subject to interference constraint at the primary receiver.

We consider one frame of duration $T$, where the fading channels are assumed unchanged. In the first phase of duration $\alpha T,(0<\alpha<1)$, SS and SR harvest energy from PBs. In the second phase $(1-\alpha) T / 2$, SS transmits its data to SR, and in the third phase $(1-\alpha) T / 2$, SR forwards the information to SD. For convenience, we denote parameters of the links between nodes in the network as in Table 1. Due to short distance between PBs and SS and SR, the line-ofsight (LoS) path is likely to exist. To this end, we assume that $h_{i s}$ and $h_{i r},(i=1,2, \ldots, N)$, follow i.n.i.d Nakagami- $m$ distribution with fading parameters $m_{i s}$ and $m_{i r}$ and squared means $\Omega_{i s}=\varepsilon\left\{\left|h_{i s}\right|^{2}\right\}$ and $\Omega_{i r}=\varepsilon\left\{\left|h_{i r}\right|^{2}\right\}$, respectively, where $\varepsilon\{\cdot\}$ denotes an expectation operation. For the other links, we assume that the fading coefficients follow i.n.i.d Rayleigh distribution.

2.1. Primary Transmitter Is Far Away from the Secondary Network. We first consider a scenario in which the primary transmitter (PT) is located far away from the secondary network (SN). Therefore, PT does not introduce interference to both SR and SD (i.e., a noise-limited scenario). Also, the 
energy harvested at SS and SD associated with the RF signal radiated by the primary transmitter is negligible.

The total energy harvested at the SS node at the end of the energy harvesting phase can be expressed as

$$
E_{s}^{\mathscr{F}}=\eta \alpha T P \sum_{i=1}^{N} L_{i s}^{0} \frac{\left|h_{i s}\right|^{2}}{d_{i s}^{\theta}}=\eta \alpha T P \sum_{i=1}^{N} L_{i s}=\eta \alpha T P \Upsilon_{s}^{\mathscr{F}},
$$

where $\eta \in(0,1)$ is the efficiency of an energy harvester, $P$ is the transmit power of $\mathrm{PB}, d_{i s}$ is the distance between the $i$ th $\mathrm{PB}$ and the source node SS, $L_{i s}^{0}$ is the path-loss at reference distance of $1 \mathrm{~m}$, and $\theta$ is the path-loss exponent. Also, $L_{i s} \triangleq$ $L_{i s}^{0}\left(\left|h_{i s}\right|^{2} / d_{i s}^{\theta}\right)$ and $\Upsilon_{s}^{\mathscr{F}} \triangleq \sum_{i=1}^{N} L_{i s}$. We note that the probability distribution function (PDF) of $L_{i s}$ is given as

$$
f\left(x, m_{i s}, \chi_{i s}\right)=\frac{x^{m_{i s}-1}}{\chi_{i s}^{m_{i s}}\left(m_{i s}-1\right) !} e^{-x / \chi_{i s}}, \quad(x>0),
$$

where $\chi_{i s} \triangleq L_{i s}^{0} \Omega_{i s} /\left(m_{i s} d_{i s}^{\theta}\right)$. Thus, the PDF of $\Upsilon_{s}^{\mathscr{F}}$ can be expressed by [29]

$$
f_{\Upsilon_{s}^{\mathscr{F}}}(y)=\sum_{i=1}^{N} \sum_{j=1}^{m_{i s}} \Phi_{s}^{\mathscr{F}}(i, j) f\left(y, j, \chi_{i s}\right),
$$

where $\Phi_{s}^{\mathscr{F}}(i, j)$ is a weighting factor defined similar to that in [29, Eq. (7)]. In particular, the recursive formula for evaluating $\Phi_{s}(i, j)$ can be expressed as

$$
\begin{aligned}
& \Phi_{s}^{\mathscr{F}}\left(i, m_{i s}\right)=\frac{\chi_{i s}^{m_{i s}}}{\prod_{h=1}^{N} \chi_{h s}^{m_{h s}}} \prod_{k=1, k \neq i}^{N}\left(\frac{1}{\chi_{k s}}-\frac{1}{\chi_{i s}}\right)^{-m_{k s}}, \\
& \Phi_{s}^{\mathscr{F}}\left(i, m_{i s}-l\right) \\
& =\frac{1}{l} \sum_{q=1, q \neq i}^{N} \sum_{k=1}^{l} \frac{m_{q s}}{\chi_{i s}^{k}}\left(\frac{1}{\chi_{i s}}-\frac{1}{\chi_{q s}}\right)^{-k} \Phi_{s}^{\mathscr{F}}\left(i, m_{i s}-l+k\right), \\
& l=1,2, \ldots, m_{i s}-1 .
\end{aligned}
$$

Similarly, the energy harvested at the SR node is given as

$$
E_{r}^{\mathscr{F}}=\eta \alpha T P \sum_{i=1}^{N} L_{i r}^{0} \frac{\left|h_{i r}\right|^{2}}{d_{i r}^{\theta}}=\eta \alpha T P Y_{r}^{\mathscr{F}},
$$

where $\Upsilon_{r}^{\mathscr{F}} \triangleq \sum_{i=1}^{N} L_{i r}^{0}\left(\left|h_{i r}\right|^{2} / d_{i r}^{\theta}\right)$ and the PDF of $\Upsilon_{r}^{\mathscr{F}}$ is expressed as

$$
f_{\Upsilon_{r}^{\mathscr{F}}}(y)=\sum_{i=1}^{N} \sum_{j=1}^{m_{i r}} \Phi_{r}^{\mathscr{F}}(i, j) f\left(y, j, \chi_{i r}\right)
$$

where $\chi_{i r} \triangleq L_{i r}^{0} \Omega_{i r} /\left(m_{i r} d_{i r}^{\theta}\right)$ and $\Phi_{r}^{\mathscr{F}}(i, j)$ is computed similar to $\Phi_{s}^{\mathscr{F}}(i, j)$.

For an underlay cognitive paradigm, the spectrum licensed to the primary network is utilized by the secondary network. However, the transmit powers of both SS and SR nodes need to be adjusted subject to the interference constraint at the primary receiver. We note that the transmit powers of SS and SR are governed by the availability of the channel state information (CSI) of the SS-PR and SR-PR links at SS and SR nodes, respectively. Thus, in this work, we consider two cases depending on whether the instantaneous CSI is available or not.

2.1.1. Instantaneous CSI of SS-PR and SR-PR Links Is Available. When the instantaneous CSI of the SS-PR and SR-PR links is available, the SS and SR can adjust their transmit powers to guarantee that the interference at $\mathrm{PR}$ is always below the maximum acceptable value $I$. Therefore, the transmit powers at SS and SR can be computed, respectively, as

$$
\begin{aligned}
P_{s}^{\mathscr{F}, \text { ins }} & =\min \left(\frac{E_{s}^{\mathscr{F}}}{(1-\alpha) T / 2}, \frac{I}{L_{s p}^{0}\left|h_{s p}\right|^{2} / d_{s p}^{\theta}}\right) \\
& =\min \left(\frac{\Upsilon_{s}^{\mathscr{F}}}{\Xi}, \frac{I}{L_{s p}}\right), \\
P_{r}^{\mathscr{F}, \text { ins }} & =\min \left(\frac{E_{r}^{\mathscr{F}}}{(1-\alpha) T / 2}, \frac{I}{L_{r p}^{0}\left|h_{r p}\right|^{2} d_{r p}^{\theta}}\right) \\
& =\min \left(\frac{\Upsilon_{r}^{\mathscr{F}}}{\Xi}, \frac{I}{L_{r p}}\right),
\end{aligned}
$$

where $\Xi \triangleq(1-\alpha) / 2 \eta \alpha P, L_{s p} \triangleq L_{s p}^{0}\left|h_{s p}\right|^{2} / d_{s p}^{\theta}$, and $L_{r p} \triangleq$ $L_{r p}^{0}\left|h_{r p}\right|^{2} / d_{r p}^{\theta}$. Given that the primary transmitter is located far away from the secondary network, we can express the SNR at $\mathrm{SR}$ and $\mathrm{SD}$ as

$$
\begin{aligned}
& \gamma_{R}^{\mathscr{F}, \text { ins }}=\min \left(\frac{\Upsilon_{s}^{\mathscr{F}}}{\Xi}, \frac{I}{L_{s p}}\right) \frac{L_{s r}}{N_{0}}, \\
& \gamma_{D}^{\mathscr{F}, \text { ins }}=\min \left(\frac{\Upsilon_{r}^{\mathscr{F}}}{\Xi}, \frac{I}{L_{r p}}\right) \frac{L_{r d}}{N_{0}},
\end{aligned}
$$

respectively, where $L_{s r} \triangleq L_{s r}^{0}\left|h_{s r}\right|^{2} / d_{s r}^{\theta}, L_{r d} \triangleq L_{r d}^{0}\left|h_{r d}\right|^{2} / d_{r d}^{\theta}$, and $N_{0}$ is the noise power of the additive white Gaussian noise (AWGN) at SR and SD. Finally, the end-to-end SNR at the SD node in the DF relay system is obtained as

$$
\gamma_{e 2 e}^{\mathscr{F} \text {,ins }}=\min \left(\gamma_{R}^{\mathscr{F}, \text { ins }}, \gamma_{D}^{\mathscr{F}, \text { ins }}\right)
$$

2.1.2. Statistical CSI of SS-PR and SR-PR Links Is Available. Let us now consider the case when only statistical CSI of the SSPR and SR-PR links is available. We note that a focus on the statistical CSI case is useful from a practical viewpoint. This is due to the fact that the collaboration between secondary nodes and PR is limited. Thus, it is hard to obtain perfect instantaneous CSI of SS-PR and SR-PR links at SS and SR, respectively. Under this condition, the interference constraint at PR due to SS transmission can be formulated as [30]

$$
\operatorname{Pr}\left\{P_{T, s} L_{s p}>I\right\} \leq \epsilon,
$$


Where Pr stands for probability, $P_{T, s}$ is the transmit power of SS, and $\epsilon$ is the tolerated error. Given that $L_{s p}$ follows an exponent distribution with mean $\chi_{s p} \triangleq L_{s p}^{0} \Omega_{s p} / d_{s p}^{\theta}$, the transmit power by SS needs to satisfy

$$
P_{T, s} \leq \frac{I}{\chi_{s p} \ln (1 / \epsilon)}
$$

Similarly, the transmit power of SR is constrained by

$$
P_{T, r} \leq \frac{I}{\chi_{r p} \ln (1 / \epsilon)}
$$

where $\chi_{r p} \triangleq L_{r p}^{0} \Omega_{r p} / d_{r p}^{\theta}$.

Thus, the actual transmit powers of SS and SR are obtained, respectively, as

$$
\begin{aligned}
& P_{s}^{\mathscr{F}, \text { ave }}=\min \left(\frac{Y_{s}^{\mathscr{F}}}{\Xi}, \frac{I}{\chi_{s p} \ln (1 / \epsilon)}\right), \\
& P_{r}^{\mathscr{F}, \text { ave }}=\min \left(\frac{\Upsilon_{r}^{\mathscr{F}}}{\Xi}, \frac{I}{\chi_{r p} \ln (1 / \epsilon)}\right) .
\end{aligned}
$$

Also, the end-to-end SNR is given as

$$
\gamma_{e 2 e}^{\mathscr{F}, \text { ave }}=\min \left(\gamma_{R}^{\mathscr{F}, \text { ave }}, \gamma_{D}^{\mathscr{F} \text {,ave }}\right)
$$

where

$$
\begin{aligned}
& \gamma_{R}^{\mathscr{F} \text {,ave }}=\min \left(\frac{\Upsilon_{s}^{\mathscr{F}}}{\Xi}, \frac{I}{\chi_{s p} \ln (1 / \epsilon)}\right) \frac{L_{s r}}{N_{0}}, \\
& \gamma_{D}^{\mathscr{F} \text {,ave }}=\min \left(\frac{\Upsilon_{r}^{\mathscr{F}}}{\Xi}, \frac{I}{\chi_{r p} \ln (1 / \epsilon)}\right) \frac{L_{r d}}{N_{0}} .
\end{aligned}
$$

2.2. Primary Transmitter Is Near to the Secondary Network. We next consider a scenario where the primary transmitter is near to the secondary network. In this case, SS and SR can further harvest energy from the RF signal radiated by PT. However, it is worth noting that PT will introduce interference to both SR and SD during the information transmission period. For analytical simplicity, we assume that the interference dominates the noises at SR and SD nodes (i.e., an interference-limited scenario).

Let $P_{0}$ denote the transmit power of the primary transmitter. In this scenario, the energy harvested by SS and SR during the energy harvesting phase consists of the energy broadcasted by PBs and the energy radiated by PT. Similar to the previous case, we can express the harvested energy at SS as

$$
\begin{aligned}
E_{s}^{\mathcal{N}} & =\eta \alpha T\left(P \sum_{i=1}^{N} L_{i s}^{0} \frac{\left|h_{i s}\right|^{2}}{d_{i s}^{\theta}}+P_{0} L_{t s}^{0} \frac{\left|h_{t s}\right|^{2}}{d_{t s}^{\theta}}\right) \\
& =\eta \alpha T P Y_{s}^{\mathcal{N}},
\end{aligned}
$$

where $\Upsilon_{s}^{\mathcal{N}} \triangleq \sum_{i=1}^{N} L_{i s}^{0}\left(\left|h_{i s}\right|^{2} / d_{i s}^{\theta}\right)+L_{t s}^{0}\left(\left|h_{t s}\right|^{2} / d_{t s}^{\theta}\left(P / P_{0}\right)\right)$. It is worth noting that $L_{t s}^{0}\left|h_{t s}\right|^{2} / d_{t s}^{\theta}\left(P / P_{0}\right)$ follows the Rayleigh distribution which can be interpreted as a Nakagami- $m$ variable with a parameter $m=1$. Therefore, $\Upsilon_{s}^{\mathcal{N}}$ is a sum of $(N+1)$ Nakagami- $m$ variables and its PDF can be evaluated in a similar manner as $\Upsilon_{s}^{\mathscr{F}}$ (cf. (6)). Also, the harvested energy at $\mathrm{SR}$ is given as

$$
\begin{aligned}
E_{r}^{\mathcal{N}} & =\eta \alpha T\left(P \sum_{i=1}^{N} L_{i r}^{0} \frac{\left|h_{i r}\right|^{2}}{d_{i r}^{\theta}}+P_{0} L_{t r}^{0} \frac{\left|h_{t r}\right|^{2}}{d_{t r}^{\theta}}\right) \\
& =\eta \alpha T P \Upsilon_{r}^{\mathcal{N}},
\end{aligned}
$$

where $Y_{r}^{\mathcal{N}} \triangleq \sum_{i=1}^{N} L_{i r}^{0}\left(\left|h_{i r}\right|^{2} / d_{i r}^{\theta}\right)+L_{t r}^{0}\left(\left|h_{t r}\right|^{2} / d_{t r}^{\theta}\left(P / P_{0}\right)\right)$. Similar to the previous scenario, the end-to-end SNR when $\mathrm{PT}$ is near to $\mathrm{SN}$ is obtained as

$$
\gamma_{e 2 e}^{\mathscr{N}}=\min \left(\gamma_{R}^{\mathscr{N}}, \gamma_{D}^{\mathscr{N}}\right)
$$

where, in the presence of the instantaneous CSI,

$$
\begin{aligned}
& \gamma_{R}^{\mathcal{N} \text {,ins }}=\min \left(\frac{\Upsilon_{s}^{\mathcal{N}}}{\Xi}, \frac{I}{L_{s p}}\right) \frac{L_{s r}}{P_{0} L_{t r}}, \\
& \gamma_{D}^{\mathcal{N} \text {,ins }}=\min \left(\frac{\Upsilon_{r}^{\mathcal{N}}}{\Xi}, \frac{I}{L_{r p}}\right) \frac{L_{r d}}{P_{0} L_{t d}},
\end{aligned}
$$

where $L_{t r} \triangleq L_{t r}^{0}\left|h_{t r}\right|^{2} / d_{t r}^{\theta}$ and $L_{t d} \triangleq L_{t d}^{0}\left|h_{t d}\right|^{2} / d_{t d}^{\theta}$. Also, when only statistical CSI is available, we have

$$
\begin{aligned}
& \gamma_{R}^{\mathcal{N} \text {,ave }}=\min \left(\frac{\Upsilon_{s}^{\mathcal{N}}}{\Xi}, \frac{I}{\chi_{s p} \ln (1 / \epsilon)}\right) \frac{L_{s r}}{P_{0} L_{t r}}, \\
& \gamma_{D}^{\mathcal{N} \text {,ave }}=\min \left(\frac{\Upsilon_{r}^{\mathcal{N}}}{\Xi}, \frac{I}{\chi_{r p} \ln (1 / \epsilon)}\right) \frac{L_{r d}}{P_{0} L_{t d}} .
\end{aligned}
$$

\section{Analysis of Power Outage Probability}

In RF-powered wireless communication systems, the received power at $\mathrm{EH}$-enabled devices must be larger than the minimum power threshold $\xi$ to activate the energy harvesting circuitry. A typical value of $\xi$ is in the range from $-30 \mathrm{dBm}$ to $-10 \mathrm{dBm}$ in practice [1]. In the considered system, if the received power is below $\xi$, the source node and relay node are inactive and thus no information transmission can occur. Thus, in this section, we evaluate the power outage probability of the system, that is, the probability that the harvested power is smaller than $\xi$.

Let us first consider the scenario where PT is far away from SN. The received power at the source node and relay node can be expressed as $P_{R}^{X}=\eta T P \Upsilon_{X}^{\mathscr{F}}$, where $X \in\{s, r\}$ (cf. (1) and (5)). Thus, the power outage probability due to insufficient harvested power at SS is calculated as

$$
p_{\text {out }, X}^{\mathscr{F}}(\xi)=\operatorname{Pr}\left(P_{R}^{X}<\xi\right)=F_{Y_{X}^{\mathscr{F}}}\left(\frac{\xi}{\eta T P}\right) \text {, }
$$


where $F_{Y_{X}^{\mathscr{F}}}(\cdot)$ is the cumulative distribution function (CDF) of $\Upsilon_{X}^{\mathscr{F}}$ that can be obtained based on (2) and (3). Consequently, (25) can be further expressed as

$$
\begin{array}{r}
P_{\text {out }, X}^{\mathscr{F}}(\xi) \\
=1-\sum_{i=1}^{N} \sum_{j=1}^{m_{i X}} \Phi_{X}^{\mathscr{F}}(i, j) e^{-\xi / \eta T P \chi_{i X}} \sum_{k=0}^{j-1} \frac{1}{k !}\left(\frac{\xi}{\eta T P \chi_{i X}}\right)^{k}, \\
X \in\{s, r\} .
\end{array}
$$

The power outage due to the insufficient harvested power in the system happens when SS or SR or both of them experience the power outage. Consequently, the power outage probability of the SN network can be computed as

$$
\begin{aligned}
p_{\text {out }, \mathrm{EH}}^{\mathscr{F}} & =1-\left[1-p_{\mathrm{out}, s}^{\mathscr{F}}(\xi)\right]\left[1-p_{\mathrm{out}, r}^{\mathscr{F}}(\xi)\right] \\
& =p_{\mathrm{out}, s}^{\mathscr{F}}(\xi)+p_{\mathrm{out}, r}^{\mathscr{F}}(\xi)-p_{\mathrm{out}, r}^{\mathscr{F}}(\xi) p_{\mathrm{out}, r}^{\mathscr{F}}(\xi) .
\end{aligned}
$$

In case that $\mathrm{PT}$ is near to $\mathrm{SN}$, we obtain a similar expression for the power outage probability; that is,

$$
p_{\text {out }, \mathrm{EH}}^{\mathcal{N}}=p_{\mathrm{out}, s}^{\mathcal{N}}(\xi)+p_{\mathrm{out}, r}^{\mathcal{N}}(\xi)-p_{\mathrm{out}, r}^{\mathcal{N}}(\xi) p_{\mathrm{out}, r}^{\mathcal{N}}(\xi),
$$

where

$$
\begin{aligned}
& p_{\text {out }, X}^{\mathcal{N}}(\xi) \\
& =1 \\
& \quad-\sum_{i=1}^{N+1} \sum_{j=1}^{m_{i X}} \Phi_{X}^{\mathcal{N}}(i, j) e^{-\xi / \eta T P \chi_{i X}} \sum_{k=0}^{j-1} \frac{1}{k !}\left(\frac{\xi}{\eta T P \chi_{i X}}\right)^{k}, \\
& X \in\{s, r\} .
\end{aligned}
$$

Numerical results based on these expressions are provided in Section 5.

\section{Analysis of Total Outage Probability}

In the considered system, the information outage at the destination node can occur due to (i) the power outage at SS and/or SR node since the harvested power is not sufficient; (ii) SS and SR being active but the channel conditions of SSSR and SR-SD links are poor so that the SNR of the received signal at the SD node is below the SNR threshold (hereafter we refer to this condition as the channel outage). Therefore, the total outage probability of the secondary network can be computed as

$$
p_{\text {out }}=p_{\text {out }, \mathrm{EH}}+\left(1-p_{\text {out }, \mathrm{EH}}\right) p_{\text {out }, \mathrm{C}}
$$

where $p_{\text {out,EH }}$ and $p_{\text {out,C }}$ are the power outage probability and the channel outage probability, respectively. In what follows, we derive expressions for the channel outage probability in two scenarios subjecting to the location of $\mathrm{PT}$ relative to the SN network.
4.1. Primary Transmitter Is Far Away from the Secondary Network. The channel outage probability of the secondary DF relay system can be expressed as [9]

$$
p_{\mathrm{out}, C}^{\mathscr{F}}=F_{\gamma_{R}^{\mathscr{F}}}\left(\gamma_{\mathrm{th}}\right)+F_{\gamma_{D}^{\mathscr{F}}}\left(\gamma_{\mathrm{th}}\right)-F_{\gamma_{R}^{\mathscr{F}}}\left(\gamma_{\mathrm{th}}\right) F_{\gamma_{D}^{\mathscr{F}}}\left(\gamma_{\mathrm{th}}\right)
$$

where $\gamma_{\text {th }}$ is the SNR threshold and $F_{\gamma_{R}^{F}}(\cdot)$ and $F_{\gamma_{D}^{F}}(\cdot)$ are the CDF of $\gamma_{R}^{\mathscr{F}}$ and $\gamma_{D}^{\mathscr{F}}$, respectively.

4.1.1. Instantaneous CSI. Let us first consider the case in which the instantaneous CSI of the SS-PR and SR-PR links is available. We have the following results.

Proposition 1. The exact CDF expression of $\gamma_{R}^{\mathscr{F}, \text { ins }}$ when the instantaneous CSI of SS-PR and SR-PR links is available is shown in (32), where $\chi_{s r}=L_{s r}^{0} \Omega_{s r} / d_{s r}^{\theta}$, and $K_{v}(\cdot)$ is the vth order modified Bessel function of the second kind [31, Eq. (8.407.1)].

$$
\begin{aligned}
& F_{\gamma_{R}^{\mathscr{F} \text {,ins }}}\left(\gamma_{t h}\right)=1-\sum_{i=1}^{N} \sum_{j=1}^{m_{i s}} \Phi_{s}^{\mathscr{F}}(i, j) \\
& \times\left\{\frac { 2 } { ( j - 1 ) ! } \left[\left(\frac{\gamma_{t h} \Xi N_{0}}{\chi_{s r} \chi_{i s}}\right)^{j / 2} K_{j}\left(2 \sqrt{\frac{\gamma_{t h} \Xi N_{0}}{\chi_{s r} \chi_{i s}}}\right)\right.\right. \\
& +\left(\frac{I \Xi}{\chi_{s p} \chi_{i s}}\right)^{j / 2} K_{j}\left(2 \sqrt{\frac{I \Xi}{\chi_{s p} \chi_{i s}}}\right)-\left(\frac{\gamma_{t h} \Xi N_{0}}{\chi_{s r} \chi_{i s}}\right. \\
& \left.\left.+\frac{I \Xi}{\chi_{s p} \chi_{i s}}\right)^{j / 2} K_{j}\left(2 \sqrt{\frac{\gamma_{t h} \Xi N_{0}}{\chi_{s r} \chi_{i s}}+\frac{I \Xi}{\chi_{s p} \chi_{i s}}}\right)\right] \\
& -\frac{I \Xi}{\chi_{s p} \chi_{i s}} \sum_{k=0}^{j-1} \frac{2}{k !}\left[\left(\frac{I \Xi}{\chi_{s p} \chi_{i s}}\right)^{(k-1) / 2}\right. \\
& \times K_{k-1}\left(2 \sqrt{\frac{I \Xi}{\chi_{s p} \chi_{i s}}}\right)-\left(\frac{\gamma_{t h} \Xi N_{0}}{\chi_{s r} \chi_{i s}}+\frac{I \Xi}{\chi_{s p} \chi_{i s}}\right)^{(k-1) / 2} \\
& \left.\left.\times K_{k-1}\left(2 \sqrt{\frac{\gamma_{t h} \Xi N_{0}}{\chi_{s r} \chi_{i s}}+\frac{I \Xi}{\chi_{s p} \chi_{i s}}}\right)\right]\right\}
\end{aligned}
$$

Proof. See Appendix A.

Based on the result in Proposition 1, we can easily deduce the CDF of $\gamma_{D}^{\mathscr{F} \text {,ins }}$ by replacing the parameters in $\gamma_{R}^{\mathscr{F} \text {,ins }}$ by their counterparts in $\gamma_{D}^{\mathscr{F} \text {,ins }}$ (i.e., $m_{i s} \rightarrow m_{i r}, \Phi_{s}^{\mathscr{F}}(i, j) \rightarrow \Phi_{r}^{\mathscr{F}}(i, j)$, $\chi_{i s} \rightarrow \chi_{i r}, \chi_{s p} \rightarrow \chi_{r p}$, and $\left.\chi_{s r} \rightarrow \chi_{r d}\right)$. Then, by substituting the CDF expressions of $\gamma_{R}^{\mathscr{F} \text {,ins }}$ and $\gamma_{D}^{\mathscr{F} \text {,ins }}$ into (31), we obtain the closed-form expression of the channel outage probability.

Asymptotic Analysis. In the high SNR regime (i.e., $P \rightarrow$ $+\infty$ or, equivalently, $\Xi \rightarrow 0$ ) and assuming that $I$ is fixed 
and independent of $P, \gamma_{R}^{\mathscr{F}}$ and $\gamma_{D}^{\mathscr{F}}$ in (8) and (9) can be approximated to

$$
\begin{aligned}
\gamma_{R}^{\infty, \mathscr{F}, \text { ins }} & =\frac{I}{L_{s p}} \frac{L_{s r}}{N_{0}}, \\
\gamma_{D}^{\infty, \mathscr{F}, \text { ins }} & =\frac{I}{L_{r p}} \frac{L_{r d}}{N_{0}} .
\end{aligned}
$$

By evaluating $\operatorname{Pr}\left(\gamma_{R}^{\infty, \mathscr{F} \text {,ins }}<\gamma_{\text {th }}\right)$ and $\operatorname{Pr}\left(\gamma_{D}^{\infty, \mathscr{F} \text {,ins }}<\gamma_{\text {th }}\right)$, we obtain the following result.

Corollary 2. The asymptotic CDFs of $\gamma_{R}^{\mathscr{F} \text {,ins }}$ and $\gamma_{D}^{\mathscr{F} \text {,ins }}$ at the high SNR regime when I is independent of $P$ are, respectively, given as

$$
\begin{aligned}
& F_{\gamma_{R}^{F, i n s}}^{\infty}\left(\gamma_{t h}\right)=\frac{\gamma_{t h} N_{0} \chi_{s p}}{\gamma_{t h} N_{0} \chi_{s p}+I \chi_{s r}}, \\
& F_{\gamma_{D}^{F, i n s}}^{\infty}\left(\gamma_{t h}\right)=\frac{\gamma_{t h} N_{0} \chi_{r p}}{\gamma_{\text {th }} N_{0} \chi_{r p}+I \chi_{r d}} .
\end{aligned}
$$

Proof. The proof is straightforward and is thus omitted to save space.

In cases that the interference threshold $I$ is proportional to transmit power $P$, that is, $I=\lambda P$, where $\lambda$ is a positive constant, we obtain the following asymptotic result.

Corollary 3. The asymptotic expression for the CDF of $\gamma_{R}^{\mathscr{F} \text {,ins }}$ at the high SNR when I is proportional to $P$ is given as

$$
\begin{aligned}
& F_{\gamma_{R}^{F}, \text { ins }}^{\infty}\left(\gamma_{t h}\right)=\frac{1}{P} \times \frac{(1-\alpha) \gamma_{t h} N_{0}}{2 \eta \alpha \chi_{s r}}\left(\sum_{i=1}^{N} \sum_{j=2}^{m_{i s}} \frac{\Phi_{s}^{\mathscr{F}}(i, j)}{(j-1) \chi_{i s}}\right. \\
& \left.-\sum_{i=1}^{N} \frac{\Phi_{s}^{\mathscr{F}}(i, 1)}{\chi_{i s}} \ln \left(\frac{(1-\alpha) \gamma_{t h} N_{0}}{2 \eta \alpha \chi_{s r} \chi_{i s} P}\right)\right) .
\end{aligned}
$$

Also, the asymptotic CDF expression of $\gamma_{D}^{\mathscr{F}, \text { ins }}$ is obtained by exchanging the parameters in $\gamma_{R}^{\mathscr{F} \text {,ins }}$ by their counterparts in $\gamma_{D}^{\mathscr{F}, \text { ins }}$.

Proof. See Appendix B.

It is worth noting that the condition of $I=\lambda P$ implies that the impact of the interference is nonnegligible regardless of the transmit power of secondary nodes [25].

4.1.2. Statistical CSI. For the case that only statistical CSI is available, we obtain the exact and asymptotic expressions as shown below.

Proposition 4. The exact CDF expression of $\gamma_{R}^{\mathscr{F}}$ ave is shown in (36), where $\mathfrak{Q}(a, n, b) \triangleq \int_{0}^{a} x^{n-1} e^{-x-b / x} d x$ and $\Gamma(\cdot, \cdot)$ is the upper incomplete Gamma function [31, Eq. (8.350.2)]:

$$
F_{\gamma_{R}^{\mathscr{F} \text {,ave }}}\left(\gamma_{t h}\right)=1-\sum_{i=1}^{N} \sum_{j=1}^{m_{i s}} \frac{\Phi_{s}^{\mathscr{F}}(i, j)}{(j-1) !}
$$

$$
\begin{aligned}
& \times\left[\mathfrak{Q}\left(\frac{I \Xi}{\chi_{s p} \chi_{i s} \ln (1 / \epsilon)}, j, \frac{\gamma_{t h} N_{0} \Xi}{\chi_{s r} \chi_{i s}}\right)\right. \\
& \left.+e^{-\gamma_{t h} N_{0} \chi_{s p} \ln (1 / \epsilon) / I \chi_{s r}} \Gamma\left(j, \frac{I \Xi}{\chi_{s p} \chi_{i s} \ln (1 / \epsilon)}\right)\right] .
\end{aligned}
$$

Proof. See Appendix C.

Corollary 5. The asymptotic expressions for CDFs of $\gamma_{R}^{\mathscr{F}}$,ave and $\gamma_{D}^{\mathscr{F} \text {, ave }}$ at the high SNR regime when $I$ is independent of $P$ are obtained as

$$
\begin{aligned}
& F_{\gamma_{R}^{\mathscr{F}, \text { ave }}}^{\infty}\left(\gamma_{t h}\right)=1-e^{-\gamma_{t h} N_{0} \chi_{s p} \ln (1 / \epsilon) / I \chi_{s r}}, \\
& F_{\gamma_{D}^{\mathscr{F}, \text { ave }}}^{\infty}\left(\gamma_{t h}\right)=1-e^{-\gamma_{t h} N_{0} \chi_{r p} \ln (1 / \epsilon) / I \chi_{r d}} .
\end{aligned}
$$

Proof. When $P \rightarrow \infty$ and $I$ is fixed, the above result is obtained based on the exact CDF expression in (36) by using the approximation of $\Gamma(n, x) \approx \Gamma(n)$, when $x \rightarrow 0$, and the property of $\sum_{i=1}^{N} \sum_{j=1}^{m_{i s}} \Phi_{s}^{\mathscr{F}}(i, j)=1$.

Corollary 6. The asymptotic expression for CDF of $\gamma_{R}^{\mathscr{F} \text {,ave }}$ when $I$ is proportional to $P$ is obtained as

$$
\begin{gathered}
F_{\gamma_{R}^{F, a v e}}^{\infty}\left(\gamma_{t h}\right)=\frac{1}{P} \times \frac{\gamma_{t h} N_{0}}{\chi_{s r}} \sum_{i=1}^{N} \sum_{j=1}^{m_{i s}} \frac{\Phi_{s}^{\mathscr{F}}(i, j)}{(j-1) !} \\
\times\left[\frac{1-\alpha}{2 \eta \alpha \chi_{i s}} \gamma\left(j-1, \frac{(1-\alpha) \lambda}{2 \eta \alpha \chi_{s p} \chi_{i s} \ln (1 / \epsilon)}\right)\right. \\
\left.+\frac{\chi_{s p} \ln (1 / \epsilon)}{\lambda} \Gamma\left(j, \frac{(1-\alpha) \lambda}{2 \eta \alpha \chi_{s p} \chi_{i s} \ln (1 / \epsilon)}\right)\right] .
\end{gathered}
$$

The asymptotic CDF expression of $\gamma_{D}^{\mathscr{F} \text {,ave }}$ is obtained by

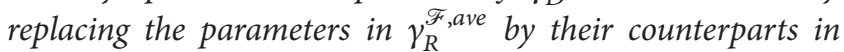
$\gamma_{D}^{\mathscr{F}}$,ave

Proof. The result is obtained by approximating the exact CDF expression in (36). Please see Appendix D for details.

4.2. Primary Transmitter Is Near to the Secondary Network. The channel outage probability of the system in this scenario can be expressed as

$$
p_{\mathrm{out}, C}^{\mathcal{N}}=F_{\gamma_{R}^{N}}\left(\gamma_{\mathrm{th}}\right)+F_{\gamma_{D}^{N}}\left(\gamma_{\mathrm{th}}\right)-F_{\gamma_{R}^{N}}\left(\gamma_{\mathrm{th}}\right) F_{\gamma_{D}^{N}}\left(\gamma_{\mathrm{th}}\right),
$$

where $F_{\gamma_{R}^{\mathcal{N}}}(\cdot)$ and $F_{\gamma_{D}^{\mathcal{N}}}(\cdot)$ are the CDFs of $\gamma_{R}^{\mathcal{N}}$ and $\gamma_{D}^{\mathcal{N}}$, respectively.

4.2.1. Instantaneous CSI. In scenarios that instantaneous CSI is available, we obtain the following results.

Proposition 7. The exact CDF expression of $\gamma_{R}^{\mathcal{N} \text {,ins }}$ is given in (40), where $\chi_{s r}=L_{s r}^{0} \Omega_{s r} / d_{s r}^{\theta}, \chi_{s p}=L_{s p}^{0} \Omega_{s p} / d_{s p}^{\theta}$, $\mathscr{A}=\gamma_{t h} \Xi P_{0} \chi_{t \mathrm{r}} /\left(\chi_{s r} \chi_{i s}\right), \mathscr{B}=I \Xi /\left(\chi_{s p} \chi_{i s}\right), \mathfrak{\Im}(a, n, b) \triangleq$ 
$\int_{a}^{\infty} x^{n / 2} e^{-b x} K_{n}(2 \sqrt{x}) d x$, and $\mathscr{W}_{k, m}(x)$ denotes the Whittaker function [31, Eq. (6.643.3)].

$$
\begin{aligned}
& F_{\gamma_{R}^{\text {N, ins }}}\left(\gamma_{t h}\right)=1-\sum_{i=1}^{N+1} \sum_{j=1}^{m_{i s}} \Phi_{s}^{\mathcal{N}}(i, j) \\
& \times\left\{j \mathscr{A}^{(j-1) / 2} e^{\mathscr{A} / 2} \mathscr{W}_{-(j+1) / 2, j / 2}(\mathscr{A})+\frac{2}{(j-1) !}\right. \\
& \times \mathscr{B}^{j / 2} K_{j}(2 \sqrt{\mathscr{B}})-\frac{2}{(j-1) !} \frac{1}{\mathscr{A}} \\
& \times e^{I \chi_{s r} / \gamma_{t h} P_{0} \chi_{s p} \chi_{t r}} \mathfrak{\Im}\left(\mathscr{B}, j, \frac{1}{\mathscr{A}}\right) \\
& -\mathscr{B}^{j-1} 2 \frac{2}{k !}\left[\mathscr{B}^{(k-1) / 2} K_{k-1}(2 \sqrt{\mathscr{B}})\right. \\
& \left.\left.-\frac{1}{\mathscr{A}} e^{I \chi_{s r} / \gamma_{t h} P_{0} \chi_{s p} \chi_{t r}} \mathfrak{\Im}\left(\mathscr{B}, k-1, \frac{1}{\mathscr{A}}\right)\right]\right\} .
\end{aligned}
$$

Proof. See Appendix E.

Corollary 8. The asymptotic CDFs of $\gamma_{R}^{\mathcal{N} \text {,ins }}$ and $\gamma_{D}^{\mathcal{N} \text {,ins }}$ at the high SNR regime when $I$ is independent of $P$ are, respectively, given as

$$
\begin{aligned}
& F_{\gamma_{R}^{N, \text { ins }}}^{\infty}\left(\gamma_{t h}\right) \\
& =1 \\
& \quad+\frac{I \chi_{s r}}{\gamma_{t h} P_{0} \chi_{s p} \chi_{t r}} e^{I \chi_{s r} / \gamma_{t h} P_{0} \chi_{s p} \chi_{t r}} E i\left(-\frac{I \chi_{s r}}{\gamma_{t h} P_{0} \chi_{s p} \chi_{t r}}\right), \\
& \quad F_{\gamma_{D}^{N, \text { ins }}}^{\infty}\left(\gamma_{t h}\right) \\
& \quad=1 \\
& \quad+\frac{I \chi_{r d}}{\gamma_{t h} P_{0} \chi_{r p} \chi_{t d}} e^{I \chi_{r d} / \gamma_{t h} P_{0} \chi_{r p} \chi_{t d}} E i\left(-\frac{I \chi_{r d}}{\gamma_{t h} P_{0} \chi_{r p} \chi_{t d}}\right),
\end{aligned}
$$

where $E i(\cdot)$ denotes the exponential integral function [31, Eq. (8.211.1)].

Proof. See Appendix F.

Corollary 9. The asymptotic CDF expression of $\gamma_{R}^{\mathcal{N} \text {,ins }}$ when $I$ is proportional to $P$ is obtained as

$$
\begin{aligned}
& F_{\gamma_{R}^{N, \text { ins }}}^{\infty}\left(\gamma_{t h}\right)=\frac{1}{P} \\
& \quad \times \frac{(1-\alpha) \gamma_{t h} P_{0} \chi_{t r}}{2 \eta \alpha \chi_{s r}}\left(\sum_{i=1}^{N+1} \sum_{j=2}^{m_{i s}} \frac{\Phi_{s}^{\mathcal{N}}(i, j)}{(j-1) \chi_{i s}}\right. \\
& \left.\quad-\sum_{i=1}^{N+1} \frac{\Phi_{s}^{\mathcal{N}}(i, 1)}{\chi_{i s}}\left[\psi(2)+\ln \left(\frac{(1-\alpha) \gamma_{t h} P_{0} \chi_{t r}}{2 \eta \alpha \chi_{s r} \chi_{i s} P}\right)\right]\right),
\end{aligned}
$$

where $\psi(\cdot)$ is the Psi function [31, Eq. (8.360.1)].

Proof. See Appendix G.
4.2.2. Statistical CSI. When only statistical CSI is available, we obtain the exact and asymptotic CDF expressions as below.

Proposition 10. The exact CDF of $\gamma_{R}^{\mathcal{N} \text {,ave }}$ is shown in (44), where $\Theta(a, n, b) \triangleq \int_{0}^{a}\left(x^{n} e^{-x} /(x+b)\right) d x$,

$$
\begin{aligned}
& F_{\gamma_{R}^{N, a v e}}\left(\gamma_{t h}\right)=1-\sum_{i=1}^{N+1} \sum_{j=1}^{m_{i s}} \frac{\Phi_{s}^{\mathcal{N}}(i, j)}{(j-1) !} \\
& \times\left[\Theta\left(\frac{I \Xi}{\chi_{s p} \chi_{i s} \ln (1 / \epsilon)}, j, \frac{\gamma_{t h} P_{0} \Xi \chi_{t r}}{\chi_{s r} \chi_{i s}}\right)\right. \\
& +\frac{I \chi_{s r}}{\gamma_{t h} P_{0} \chi_{t r} \chi_{s p} \ln (1 / \epsilon)+I \chi_{s r}} \\
& \left.\times \Gamma\left(j, \frac{I \Xi}{\chi_{s p} \chi_{i s} \ln (1 / \epsilon)}\right)\right]
\end{aligned}
$$

Proof. As a sketch of the proof, we first calculate the unconditional CDF of $F_{\gamma_{R}^{N \text {,ave }} \mid L_{t r}}\left(\gamma_{\text {th }}, L_{t r}\right)$ (cf. (23)). Then, the unconditional CDF expression is obtained by taking the expectation of $F_{\gamma_{R}^{N}, \text { ave } \mid L_{t r}}\left(\gamma_{\text {th }}, L_{t r}\right)$ with respect to $L_{t r}$.

Corollary 11. The asymptotic CDF expressions of $\gamma_{R}^{\mathcal{N} \text {,ave }}$ and $\gamma_{D}^{\mathcal{N}, \text { ave }}$ when $I$ is fixed and independent of $P$ are, respectively, given as

$$
\begin{aligned}
& F_{\gamma_{R}^{N, \text { ave }}}^{\infty}\left(\gamma_{t h}\right)=\frac{\gamma_{t h} P_{0} \chi_{t r} \chi_{s p} \ln (1 / \epsilon)}{\gamma_{t h} P_{0} \chi_{t r} \chi_{s p} \ln (1 / \epsilon)+I \chi_{s r}} \\
& F_{\gamma_{D}^{N, \text { ave }}}^{\infty}\left(\gamma_{t h}\right)=\frac{\gamma_{t h} P_{0} \chi_{t d} \chi_{r p} \ln (1 / \epsilon)}{\gamma_{t h} P_{0} \chi_{t d} \chi_{r p} \ln (1 / \epsilon)+I \chi_{r d}} .
\end{aligned}
$$

Proof. The result is obtained based on the exact CDF expression in (44) by using the approximated formula of $\Gamma(n, x) \approx$ $\Gamma(n)$, when $x \rightarrow 0$, and the property of $\sum_{i=1}^{N+1} \sum_{j=1}^{m_{i s}} \Phi_{s}^{\mathcal{N}}(i, j)=$ 1.

Corollary 12. The asymptotic CDF expression of $\gamma_{R}^{\mathcal{N} \text {,ave }}$ when $I$ is proportional to $P$ is obtained as

$$
\begin{gathered}
F_{\gamma_{R}^{\prime, ~ a v e ~}}^{\infty}\left(\gamma_{t h}\right)=\frac{1}{P} \times \frac{\gamma_{t h} P_{0} \chi_{t r}}{\chi_{s r}} \sum_{i=1}^{N+1} \sum_{j=1}^{m_{i s}} \frac{\Phi_{s}^{\mathcal{N}}(i, j)}{(j-1) !} \\
\times\left[\frac{1-\alpha}{2 \eta \alpha \chi_{i s}} \gamma\left(j-1, \frac{(1-\alpha) \lambda}{2 \eta \alpha \chi_{s p} \chi_{i s} \ln (1 / \epsilon)}\right)\right. \\
\left.+\frac{\chi_{s p} \ln (1 / \epsilon)}{\lambda} \Gamma\left(j, \frac{(1-\alpha) \lambda}{2 \eta \alpha \chi_{s p} \chi_{i s} \ln (1 / \epsilon)}\right)\right] .
\end{gathered}
$$

Proof. See Appendix H.

Remarks 1. From the derived asymptotic expressions above, we have some remarks in the high SNR regime as follows:

(1) From Corollaries 2, 5, 8, and 11, it is observed that when the interference threshold $I$ is fixed and 
independent of the transmit power $P$, an outage probability floor occurs in the high SNR regime. The larger the value $I$ is, the lower the floor appears. In addition, this outage probability behavior also implies that no diversity gain is achieved in the high SNR region under these scenarios.

(2) In scenarios that PT is near to the secondary network, a smaller transmit power of the primary transmitter results in lower outage probability. When only statistical CSI is available, a higher tolerated error $\epsilon$ also leads to lower outage probability (see, e.g., Corollary 11).

(3) When $I$ is proportional to $P$, it can be concluded from Corollaries 6 and 12 that a diversity order equal to one is attained.

Numerical results based on the derived expressions will be presented in Section 5.

\section{Results and Discussions}

In this section, we provide simulation results to validate the derived expressions in the previous section. Also, we evaluate impacts of the transmit power of power-beacons, transmit power of the primary transmitter, and the interference threshold at the primary receiver on the outage probability of the secondary relay system. We examine two scenarios, namely, Scenario 1 ( $N=3 \mathrm{PBs})$ and Scenario $2(N=5 \mathrm{PBs})$. Without loss of generality, we assume that the coordinates of the SS, SR, SD, and PR nodes in the $x y$-plane are $(0,0),(1,4)$, $(7,10)$, and $(8,2)$, respectively. For Scenario 1 , the PBs are located at $(0,2),(5,3)$, and $(-1,2)$. In Scenario 2 , the locations of the two additional PBs are at $(0,5)$ and $(-2,-2)$. In case that PT is located near the secondary network, we assume that the coordinate of PT is $(20,30)$. We consider $m_{i s}=i, m_{i r}=i+1$, $\Omega_{i s}=\Omega_{i r}=0.5 i$ for simple presentation given that listing out all the values is lengthy. Other simulation parameters are $T=1, \alpha=0.3, \eta=0.8, L_{0}=10^{-3}, \theta=3.5, \epsilon=0.1, \gamma_{\text {th }}=0 \mathrm{~dB}$, $N_{0}=10^{-11} \mathrm{~W}, \Omega_{s r}=0.5, \Omega_{s p}=0.5, \Omega_{r d}=1$, and $\Omega_{r p}=1$.

In Figure 2, we plot the power outage probability versus the transmit power of power-beacons. The power threshold for EH circuitry activation $\xi$ is set to $-30 \mathrm{dBm}$ [1]. It can be seen that when the transmit power of PBs is $20 \mathrm{dBm}$, the probability that the outage occurs due to insufficient harvested energy is very low. Moreover, when the number of PBs is increased (i.e., in Scenario 2), the power outage is reduced significantly since a larger amount of energy is harvested. Another observation is that when the primary transmitter (PT) is near to $\mathrm{SN}$, the outage probability is smaller as additional energy is harvested from PT. However, this benefit becomes less significant when the number of PBs is increased.

In Figures 3 and 4, we plot the channel outage probability versus $P$ when the instantaneous CSI is available. When there is only the statistical CSI, the results are shown in Figures 5 and 6 . It can be seen from all the figures that the curves based on analysis match exactly with the simulation curves in all cases, which confirms the accuracy of the derived expressions. Moreover, as expected, the asymptotic curves

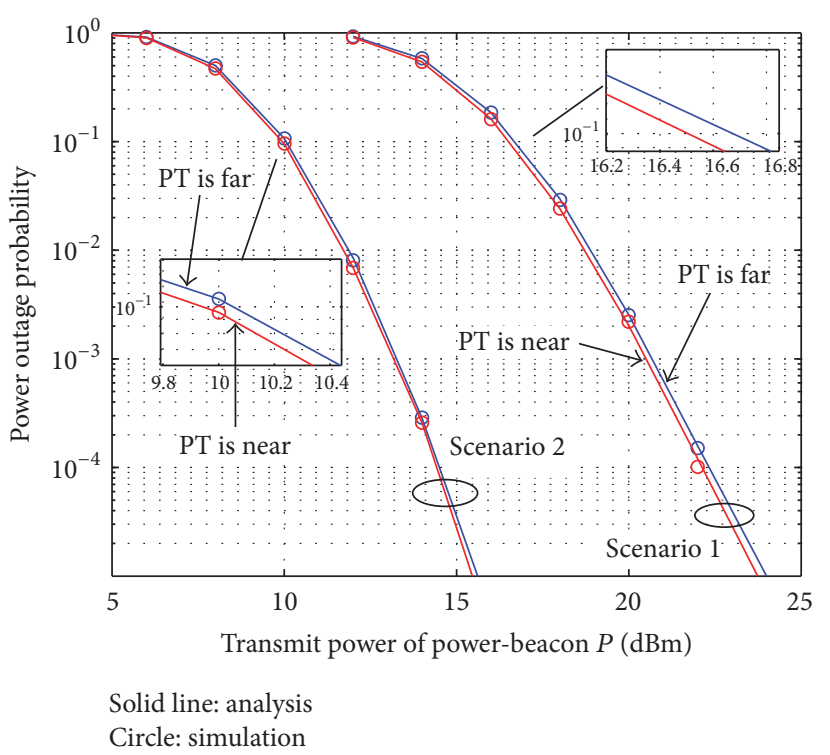

FIgURE 2: Power outage probability versus transmit power of PBs.

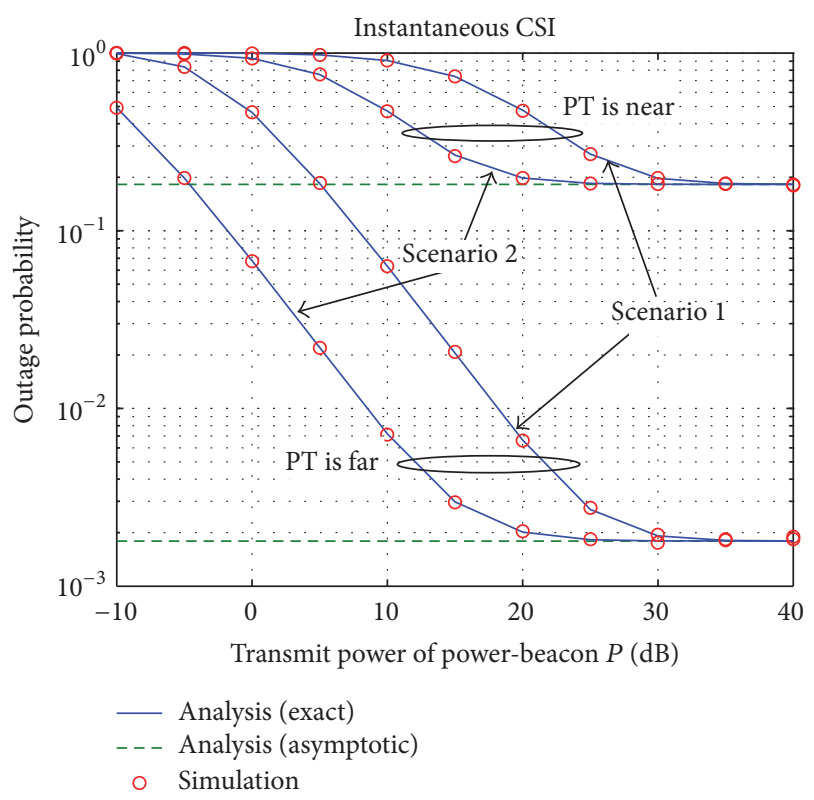

FIgURE 3: Channel outage probability versus $P\left(I=-80 \mathrm{~dB}\right.$ and $P_{0}=$ $100 \mathrm{~mW})$.

are close to the exact curves in the high SNR regime. We have some other important observations from these results as follows:

(1) In Scenario 2, the outage probability is lower than that in Scenario 1 since higher energy is harvested due to a larger number of employed PBs.

(2) When PT is located close to the secondary network, the outage probability is higher compared to its counterparts. This is because, under this condition, the benefit of increased energy harvesting at SS and SR nodes thanks to the signal radiated by PT is not 


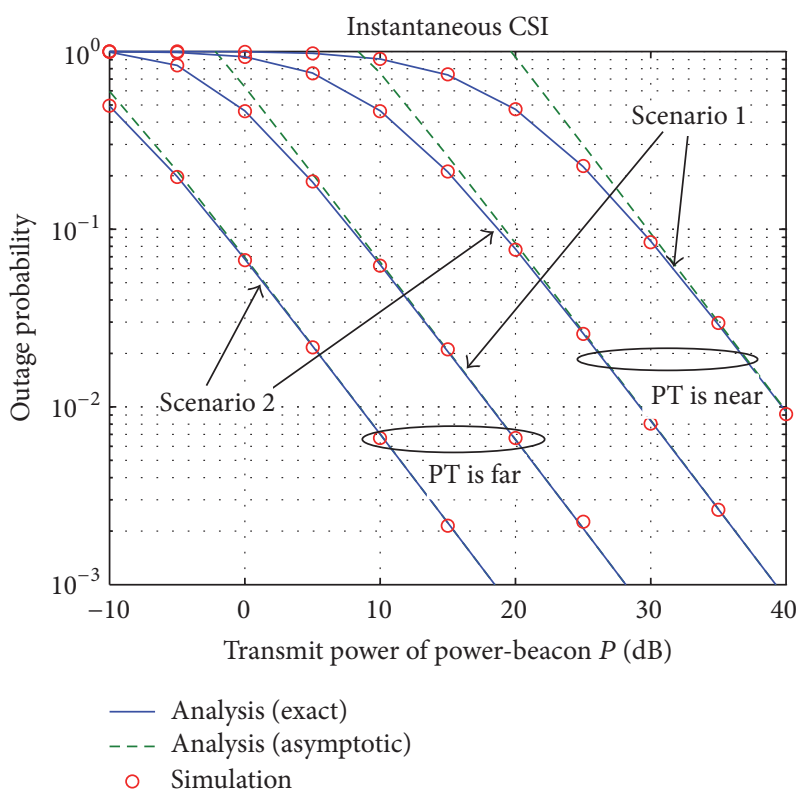

Figure 4: Channel outage probability versus $P\left(I=\lambda P, \lambda=10^{-8}\right.$, and $P_{0}=100 \mathrm{~mW}$ ).

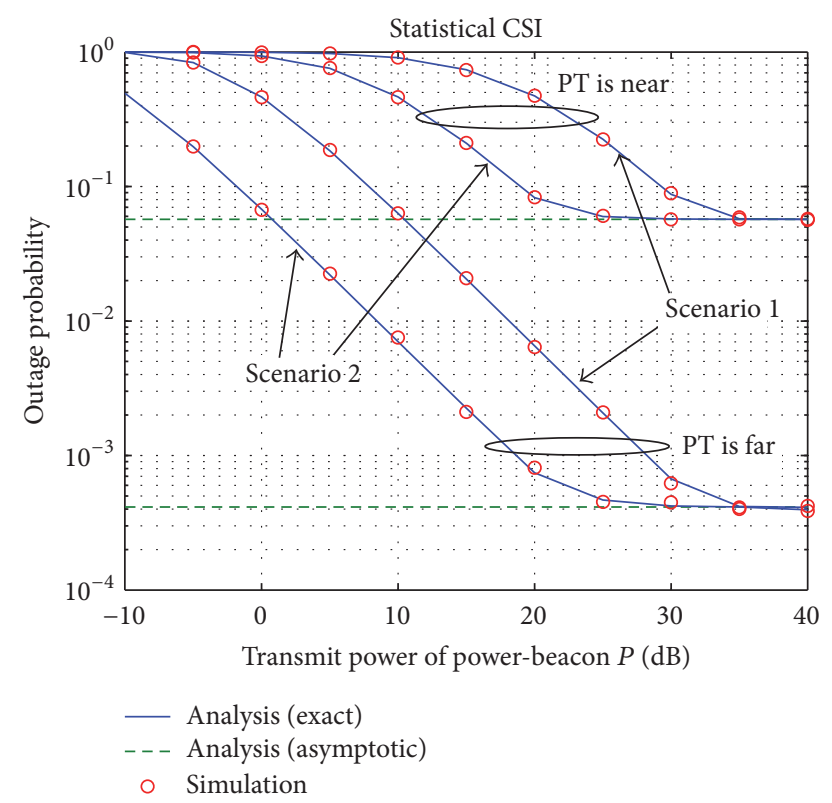

Figure 5: Channel outage probability versus $P\left(I=-70 \mathrm{~dB}\right.$ and $P_{0}=$ $100 \mathrm{~mW})$.

significant compared to the disadvantages due to the interference that this signal introduces at SR and SD nodes.

(3) When $I$ is fixed and independent of $P$, the outage probability floor appears at the high SNR region. This is due to the fact that, for a fixed value $I$, increasing $P$ does not necessarily lead to higher transmit power of SS and SR nodes since the transmit power is now determined by $I$ (cf. (7), (14)-(15)). Note that this behavior of the outage probability suggests that

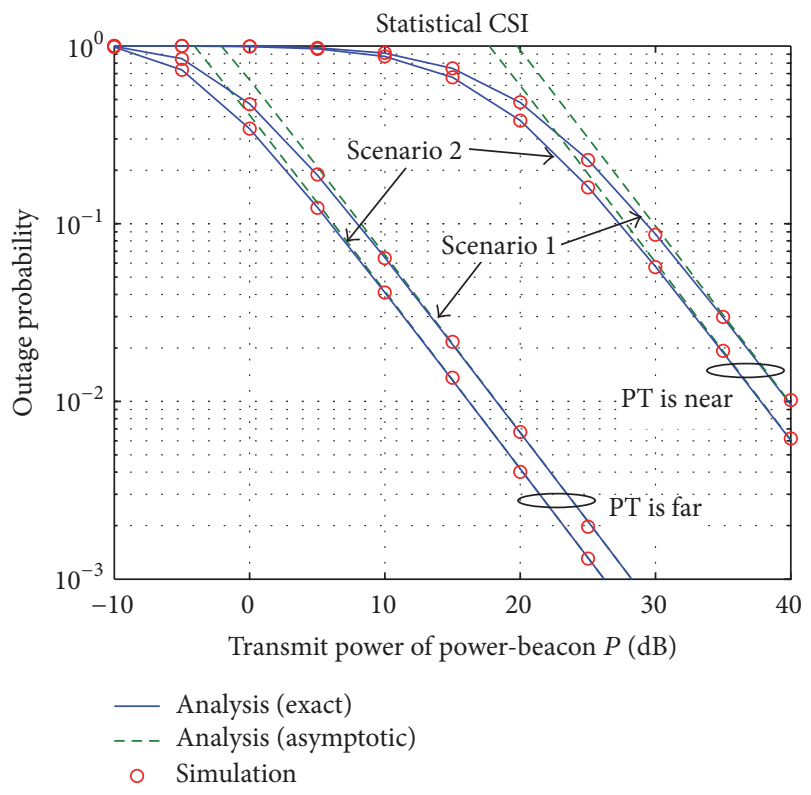

FIGURE 6: Channel outage probability versus $P\left(I=\lambda P, \lambda=10^{-10}\right.$, and $P_{0}=100 \mathrm{~mW}$ ).

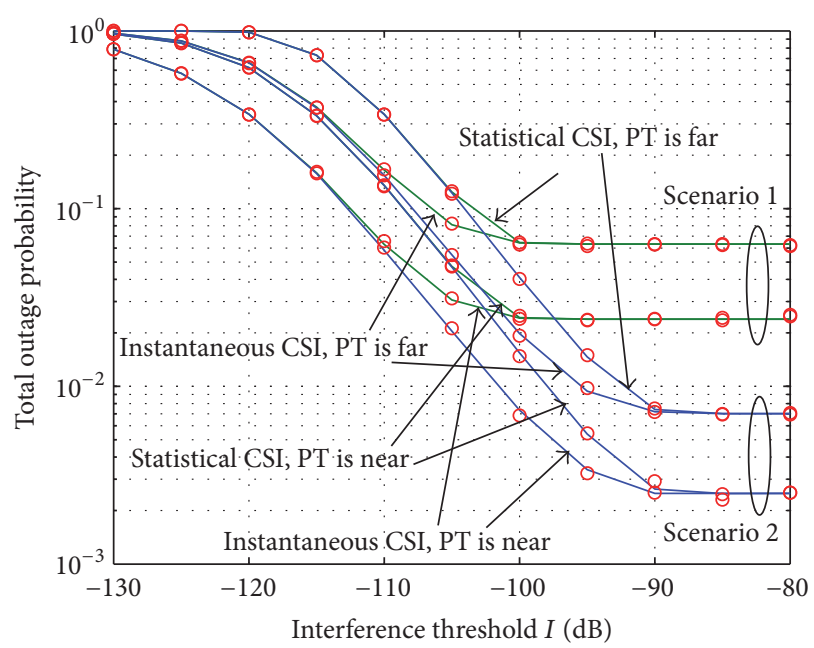

Solid line: analysis

Circle: simulation

FIGURE 7: Outage probability versus interference threshold $I\left(\gamma_{\text {th }}=\right.$ $-10 \mathrm{~dB}$ and PT is located at $(80,120))$.

cognitive relay systems are not applicable in the high SNR regime.

(4) When $I$ is proportional to $P$, a full diversity order can be attained. A similar conclusion was made in [25], where a different EH-based CRN system was investigated.

To further investigate impacts of the primary network on the outage probability of the secondary relay network, we plot in Figures 7 and 8 the total outage probability versus the tolerant interference threshold $I$ and the transmit power $P_{0}$, respectively. It can be observed from Figure 7 that the outage 


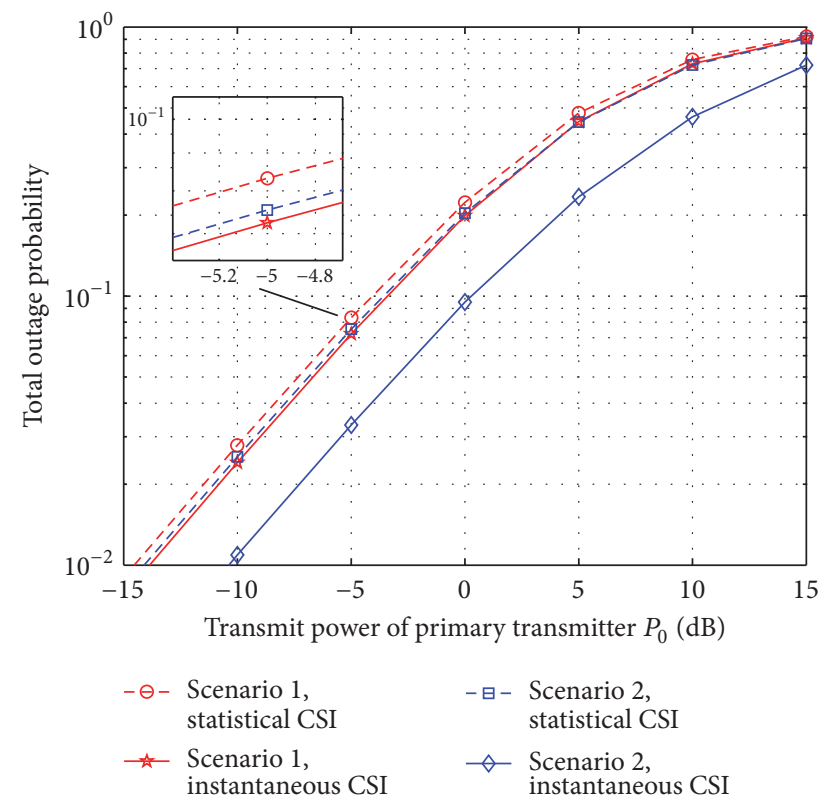

FIGURE 8: Outage probability versus transmit power of primary transmitter $P_{0}\left(\gamma_{\text {th }}=-10 \mathrm{~dB}\right.$ and $\left.P=1 \mathrm{~W}\right)$.

probability is decreased when the interference threshold $I$ is higher. This is because larger $I$ implies that higher transmit power of SS and SR can be allowed, which in turns leads to lower outage probability. However, the outage probability exhibits an error floor when $I$ is sufficiently large. This is due to the fact that, in this region, the maximum transmit power of SS and SR depends on the harvested energy only since the interference constraints are relaxed (cf. (7), (14)-(15)). This also explains why in Figure 7 the error floors in Scenario 2 are lower than those in Scenario 1. In Figure 8, we notice that higher $P_{0}$ results in higher outage probability in all scenarios. This can be explained similar to Comment 2 regarding the location of PT relative to $\mathrm{SN}$ nodes presented in the previous paragraph.

\section{Conclusions}

In this paper, we have studied distributed power-beaconassisted energy harvesting cognitive relay wireless networks. We have considered practical scenarios that (i) secondary nodes might be inactive due to insufficient harvested energy; (ii) the fading channels from PBs to secondary nodes undergo i.n.i.d Nakagami- $m$ fading distribution, and (iii) the instantaneous CSI of the links between SN nodes and PR might not be available. The exact expressions for the outage probability of the secondary network under difference scenarios have been derived. Asymptotic analysis for the outage probability has also been performed, which provides insights into the system behavior. We have evaluated the impacts of the transmit power $P$ of $\mathrm{PBs}$, the interference threshold $I$ at the primary receiver, and the transmit power $P_{0}$ of the primary transmitter on the outage probability. The results show that when $I$ is fixed and independent of $P$, the outage probability floor exhibits at the high SNR region. On the other hand, if $I$ is proportional to
$P$, a full diversity order could be achieved. Our future work would be a consideration of a general system model where nodes are equipped with multiple antennas.

\section{Appendix}

\section{A. Proof of Proposition 1}

The CDF of $\gamma_{R}^{\mathscr{F} \text {,ins }}$ can be calculated as (cf. (8))

$$
\begin{aligned}
& F_{\gamma_{R}^{F, \text { ins }}}\left(\gamma_{\text {th }}\right)=\operatorname{Pr}\left(\min \left(\frac{\Upsilon_{s}^{\mathscr{F}}}{\Xi}, \frac{I}{L_{s p}}\right) \frac{L_{s r}}{N_{0}}<\gamma_{\mathrm{th}}\right) \\
& =\operatorname{Pr}\left(\frac{\Upsilon_{s}^{\mathscr{F}}}{\Xi} \frac{L_{s r}}{N_{0}}<\gamma_{\mathrm{th}}, \frac{\Upsilon_{s}^{\mathscr{F}}}{\Xi}<\frac{I}{L_{s p}}\right) \\
& +\operatorname{Pr}\left(\frac{I}{L_{s p}} \frac{L_{s r}}{N_{0}}<\gamma_{\mathrm{th}}, \frac{\Upsilon_{s}^{\mathscr{F}}}{\Xi} \geq \frac{I}{L_{s p}}\right) \\
& =\int_{0}^{\infty} F_{L_{s r}}\left(\frac{\gamma_{\mathrm{th}} \Xi N_{0}}{x}\right) F_{L_{s p}}\left(\frac{I \Xi}{x}\right) f_{\Upsilon_{s}^{\mathscr{F}}}(x) d x \\
& +\int_{0}^{\infty} F_{L_{s r}}\left(\frac{\gamma_{\mathrm{th}} N_{0} x}{I}\right)\left[1-F_{\Upsilon_{s}^{\mathscr{F}}}\left(\frac{I \Xi}{x}\right)\right] \\
& \quad \times f_{L_{s p}}(x) d x .
\end{aligned}
$$

Note that $L_{s r}$ and $L_{s p}$ are exponentially distributed random variables with parameters of $\chi_{s r}=L_{s r}^{0} \Omega_{s r} / d_{s r}^{\theta}$ and $\chi_{s p}=$ $L_{s p}^{0} \Omega_{s p} / d_{s p}^{\theta}$, respectively (i.e., $F_{L_{s r}}(x)=1-e^{-x / \chi_{s r}}, F_{L_{s p}}(x)=$ $1-e^{-x / \chi_{s p}}$, and $\left.f_{L_{s p}}(x)=\left(1 / \chi_{s p}\right) e^{-x / \chi_{s p}}, x>0\right)$. Also, the CDF of $Y_{s}^{\mathscr{F}}$ can be obtained from the PDF function in (3) as

$$
F_{Y_{s}^{\mathscr{F}}}(y)=1-\sum_{i=1}^{N} \sum_{j=1}^{m_{i s}} \Phi_{s}^{\mathscr{F}}(i, j) e^{-y / \chi_{i s}} \sum_{k=0}^{j-1} \frac{1}{k !}\left(\frac{y}{\chi_{i s}}\right)^{k} .
$$

By substituting these results into (A.1) and applying the integral results in [31, Eq. (3.478.4)] and [31, Eq. (3.351.3)], we obtain (32) after some mathematical manipulations.

\section{B. Proof of Corollary 3}

When $P \rightarrow+\infty$ and $I=\lambda P$, we note that $\gamma_{\mathrm{th}} \Xi N_{0} /\left(\chi_{s r} \chi_{i s}\right) \rightarrow$ 0 and $I \Xi /\left(\chi_{s p} \chi_{i s}\right)=(1-\alpha) \lambda /\left(2 \eta \alpha \chi_{s p} \chi_{i s}\right)$ is a constant. Thus, the second and the third terms within the summation in (32) cancel out with each other and are similar to the fourth and the fifth terms. Consequently, the exact CDF expression in (32) can be approximated to

$$
\begin{aligned}
& F_{\gamma_{R}^{F}} \text {,ins } \\
& \quad\left(\gamma_{\mathrm{th}}\right)=1-\sum_{i=1}^{N} \sum_{j=1}^{m_{i s}} \Phi_{s}^{\mathscr{F}}(i, j) \\
& \quad \times \frac{2}{(j-1) !}\left(\frac{\gamma_{\mathrm{th}} \Xi N_{0}}{\chi_{s r} \chi_{i s}}\right)^{j / 2} K_{j}\left(2 \sqrt{\frac{\gamma_{\mathrm{th}} \Xi N_{0}}{\chi_{s r} \chi_{i s}}}\right) .
\end{aligned}
$$


On the other hand, it can be proved from (2) and (3) that

$$
\sum_{i=1}^{N} \sum_{j=1}^{m_{i s}} \Phi_{s}^{\mathscr{F}}(i, j)=1
$$

Therefore, by using the series representation of the Bessel function $K_{v}(z)$ provided in [31, Eq. (8.446)], we can approximate (B.1) to (35) after some manipulations.

\section{Proof of Proposition 4}

From (17), we can evaluate the CDF of $\gamma_{R}^{\mathscr{F} \text {,ave }}$ as

$$
\begin{aligned}
F_{\gamma_{R}^{\mathscr{F} \text { ave }}} & \left(\gamma_{\mathrm{th}}\right) \\
= & \operatorname{Pr}\left(\min \left(\frac{\Upsilon_{s}^{\mathscr{F}}}{\Xi}, \frac{I}{\chi_{s p} \ln (1 / \epsilon)}\right) \frac{L_{s r}}{N_{0}}<\gamma_{\mathrm{th}}\right) \\
= & \operatorname{Pr}\left(L_{s r}<\frac{\gamma_{\mathrm{th}} N_{0} \Xi}{\Upsilon_{s}^{\mathscr{F}}}, \Upsilon_{s}^{\mathscr{F}}<\frac{I \Xi}{\chi_{s p} \ln (1 / \epsilon)}\right) \\
& +\operatorname{Pr}\left(L_{s r}<\frac{\gamma_{\mathrm{th}} N_{0} \chi_{s p} \ln (1 / \epsilon)}{I}\right) \\
& \times \operatorname{Pr}\left(\Upsilon_{s}^{\mathscr{F}} \geq \frac{I \Xi}{\chi_{s p} \ln (1 / \epsilon)}\right) \\
= & \int_{0}^{I \Xi / \chi_{s p} \ln (1 / \epsilon)} F_{L_{s r}\left(\frac{\gamma_{\mathrm{th}} \Xi N_{0}}{x}\right) f_{\Upsilon_{s}^{\mathscr{F}}}(x) d x} \\
& +F_{L_{s r}}\left(\frac{\gamma_{\mathrm{th}} N_{0} \chi_{s p} \ln (1 / \epsilon)}{I}\right) \\
& \times\left[1-F_{\Upsilon_{s}^{\mathscr{F}}}\left(\frac{I \Xi}{\chi_{s p} \ln (1 / \epsilon)}\right)\right] .
\end{aligned}
$$

By substituting the expressions of $F_{L_{s p}}(x), F_{L_{s r}}(x), f_{\Upsilon_{s}^{F}}(\cdot)$, and $F_{Y_{s}^{\mathscr{F}}}(\cdot)$ into (C.1) and then using the results in [31, Eq. (3.351.1)], [31, Eq. (8.352.6)], and [31, Eq. (8.352.7)], we finally obtain (36).

\section{Proof of Corollary 6}

By adopting the approximation of $e^{-x} \approx 1-x$, when $x \rightarrow 0$, we can approximate the integral $\mathfrak{Q}(a, n, b)$ defined in Proposition 4 when $b \rightarrow 0$ as

$$
\begin{aligned}
\mathfrak{Q}(a, n, b) & \triangleq \int_{0}^{a} x^{n-1} e^{-x-b / x} d x \\
& \approx \int_{0}^{a} x^{n-1} e^{-x}\left(1-\frac{b}{x}\right) d x \\
& =\gamma(n, a)-b \gamma(n-1, a),
\end{aligned}
$$

where $\gamma(n, a)$ is the lower incomplete Gamma function [31, Eq. (8.350.1)]. Recall that $\Xi \triangleq(1-\alpha) /(2 \eta \alpha P)$ and $I=\lambda P$. Thus, we have $I \Xi=(1-\alpha) \lambda /(2 \eta \alpha)$ that is a constant. Thus, under the condition that $P \rightarrow \infty$ and $I=\lambda P$, the exact CDF expression in (36) can be approximated to

$$
\begin{aligned}
& F_{\gamma_{R}^{F}, \text { ave }}\left(\gamma_{\text {th }}\right) \approx 1-\sum_{i=1}^{N} \sum_{j=1}^{m_{i s}} \frac{\Phi_{s}^{\mathscr{F}}(i, j)}{(j-1) !} \\
& \times\left[\gamma\left(j, \frac{I \Xi}{\chi_{s p} \chi_{i s} \ln (1 / \epsilon)}\right)-\frac{\gamma_{\mathrm{th}} N_{0} \Xi}{\chi_{s p} \chi_{i s}}\right. \\
& \times \gamma\left(j-1, \frac{I \Xi}{\chi_{s p} \chi_{i s} \ln (1 / \epsilon)}\right) \\
& +\left(1-\frac{\gamma_{\mathrm{th}} N_{0} \chi_{s p} \ln (1 / \epsilon)}{I \chi_{s r}}\right) \\
& \left.\quad \times \Gamma\left(j, \frac{I \Xi}{\chi_{s p} \chi_{i s} \ln (1 / \epsilon)}\right)\right]
\end{aligned}
$$

By using the equation of $\gamma(n, a)+\Gamma(n, a)=\Gamma(n)$ and the property of $\sum_{i=1}^{N} \sum_{j=1}^{m_{i s}} \Phi_{s}^{\mathscr{F}}(i, j)=1$, we can simplify (D.2) to (38) after some manipulations. This completes the proof.

\section{E. Proof of Proposition 7}

Let us begin by calculating the conditional CDF of $F_{\gamma_{R}^{\mathcal{N} \text {,ins } \mid L_{t r}}}\left(\gamma_{\mathrm{th}}, L_{t r}\right)$ with respect to $L_{t r}$, that is,

$$
\begin{aligned}
& F_{\gamma_{R}^{\prime, \text { ins }} \mid L_{t r}}\left(\gamma_{\mathrm{th}}, L_{t r}\right) \\
& \quad=\operatorname{Pr}\left(\min \left(\frac{\Upsilon_{s}^{\mathcal{N}}}{\Xi}, \frac{I}{L_{s p}}\right) \frac{L_{s r}}{P_{0} L_{t r}}<\gamma_{\text {th }}\right) .
\end{aligned}
$$

We note that there is the similarity in terms of mathematical description between (A.1) and (E.1) (i.e., $N_{0}$ versus $P_{0} L_{t r}$ ). Thus, similar to the proof in Appendix A, we can express the closed-form expression for $F_{\gamma_{R}^{N, \text { ins }} \mid L_{t r}}\left(\gamma_{\mathrm{th}}, L_{t r}\right)$ as in (E.2).

$$
\begin{aligned}
& F_{\gamma_{R}^{N, \text { ins }} \mid L_{t r}}\left(\gamma_{\mathrm{th}}, L_{t r}\right)=1-\sum_{i=1}^{N+1} \sum_{j=1}^{m_{i s}} \Phi_{s}^{\mathcal{N}}(i, j) \\
& \times\left\{\frac { 2 } { ( j - 1 ) ! } \left[\left(\frac{\gamma_{\mathrm{th}} \Xi P_{0} L_{t r}}{\chi_{s r} \chi_{i s}}\right)^{j / 2}\right.\right. \\
& \times K_{j}\left(2 \sqrt{\frac{\gamma_{\mathrm{th}} \Xi P_{0} L_{t r}}{\chi_{s r} \chi_{i s}}}\right)+\left(\frac{I \Xi}{\chi_{s p} \chi_{i s}}\right)^{j / 2} \\
& \times K_{j}\left(2 \sqrt{\frac{I \Xi}{\chi_{s p} \chi_{i s}}}\right)-\left(\frac{\gamma_{\mathrm{th}} \Xi P_{0} L_{t r}}{\chi_{s r} \chi_{i s}}+\frac{I \Xi}{\chi_{s p} \chi_{i s}}\right)^{j / 2} \\
& \left.\times K_{j}\left(2 \sqrt{\frac{\gamma_{\mathrm{th}} \Xi P_{0} L_{t r}}{\chi_{s r} \chi_{i s}}+\frac{I \Xi}{\chi_{s p} \chi_{i s}}}\right)\right]-\frac{I \Xi}{\chi_{s p} \chi_{i s}} \sum_{k=0}^{j-1} \frac{2}{k !} \\
& \times\left[\left(\frac{I \Xi}{\chi_{s p} \chi_{i s}}\right)^{(k-1) / 2} \times K_{k-1}\left(2 \sqrt{\frac{I \Xi}{\chi_{s p} \chi_{i s}}}\right)\right.
\end{aligned}
$$




$$
\begin{aligned}
& -\left(\frac{\gamma_{\mathrm{th}} \Xi P_{0} L_{t r}}{\chi_{s r} \chi_{i s}}+\frac{I \Xi}{\chi_{s p} \chi_{i s}}\right)^{(k-1) / 2} \\
& \left.\left.\times K_{k-1}\left(2 \sqrt{\frac{\gamma_{\mathrm{th}} \Xi P_{0} L_{t r}}{\chi_{s r} \chi_{i s}}+\frac{I \Xi}{\chi_{s p} \chi_{i s}}}\right)\right]\right\} .
\end{aligned}
$$

The unconditional CDF of $\gamma_{R}^{\mathcal{N} \text {,ins }}$ is then obtained by taking the expectation of $F_{\gamma_{R}^{, V} \text {,ins } \mid L_{t r}}\left(\gamma_{\text {th }}, L_{t r}\right)$ with respect to $L_{t r}$. In particular, we have

$$
F_{\gamma_{R}^{\mathcal{N} \text {,ins }}}\left(\gamma_{\text {th }}\right)=\int_{0}^{\infty} F_{\gamma_{R}^{\mathcal{N} \text {,ins }} \mid L_{t r}}\left(\gamma_{\text {th }}, x\right) f_{L_{t r}}(x) d x,
$$

where $f_{L_{t r}}(\cdot)$ is the PDF of $L_{t r}$, that is, $f_{L_{t r}}(x)=(1 /$ $\left.\chi_{t r}\right) e^{-x / \chi_{t r}}(x>0)$. By applying the integral result of $\int_{0}^{+\infty} t^{\epsilon-1 / 2} e^{-\alpha t} K_{2 \nu}(2 \beta \sqrt{t}) d t=\left(\alpha^{-\epsilon} / 2 \beta\right) e^{\beta^{2} / 2 \alpha} \mathscr{W}_{-\epsilon, \nu}\left(\beta^{2} /\right.$ $\alpha) \Gamma(\epsilon-\nu+1 / 2) \Gamma(\epsilon+\nu+1 / 2)$, where $\mathscr{W}$ denotes the Whittaker function [31, Eq. (6.643.3)] and $\Gamma(\cdot)$ is the Gamma function [31, Eq. (8.310.1)], the integral in (E.3) can be solved. The result in $(40)$ is thus obtained.

\section{F. Proof of Corollary 8}

In the high SNR regime (i.e., $\Xi \rightarrow 0$ ), $\gamma_{R}^{\mathcal{N}}$ defined in (14) can be approximated to

$$
\gamma_{R}^{\infty, \mathcal{N}}=\frac{I}{L_{s p}} \frac{L_{s r}}{P_{0} L_{t r}} .
$$

The CDF of $\gamma_{R}^{\infty, N}$ conditioned on $L_{t r}$ can be computed as

$$
\begin{aligned}
F_{\gamma_{R}^{\infty, N} \mid L_{t r}}\left(\gamma_{\mathrm{th}}\right) & =\operatorname{Pr}\left(\frac{I}{L_{s p}} \frac{L_{s r}}{P_{0} L_{t r}}<\gamma_{\mathrm{th}}\right) \\
& =\operatorname{Pr}\left(L_{s r}<\frac{\gamma_{\mathrm{th}} P_{0} L_{t r} L_{s p}}{I}\right) \\
& =\int_{0}^{\infty} F_{L_{s r}}\left(\frac{\gamma_{\mathrm{th}} P_{0} L_{t r} x}{I}\right) f_{L_{s p}}(x) d x \\
& =\int_{0}^{\infty}\left[1-e^{-\gamma_{\mathrm{th}} P_{0} L_{t r} x / I \chi_{s r}}\right] \frac{1}{\chi_{s p}} e^{-x / \chi_{s p}} d x \\
& =\frac{\gamma_{\mathrm{th}} P_{0} \chi_{s p} L_{t r}}{\gamma_{\mathrm{th}} P_{0} \chi_{s p} L_{t r}+I \chi_{s r}} .
\end{aligned}
$$

The unconditional CDF of $\gamma_{R}^{\infty, \mathcal{N}}$ is then obtained as

$$
\begin{aligned}
F_{\gamma_{R}^{\infty, N}}\left(\gamma_{\mathrm{th}}\right) & =\int_{0}^{\infty} F_{\gamma_{R}^{\infty, N} \mid L_{t r}}\left(\gamma_{\mathrm{th}}, x\right) f_{L_{t r}}(x) d x \\
& =\int_{0}^{\infty} \frac{\gamma_{\mathrm{th}} P_{0} \chi_{s p} x}{\gamma_{\mathrm{th}} P_{0} \chi_{s p} x+I \chi_{s r}} \frac{1}{\chi_{t r}} e^{-x / \chi_{\text {tr }}} d x .
\end{aligned}
$$

By using the integral result in [31, Eq. (3.353.5)], we can solve the integral in (F.3), which finally yields (41). In addition, the CDF expression of $\gamma_{D}^{\infty, N}$ is obtained by exchanging the parameters in $\gamma_{R}^{\infty, N}$ by its counterparts in $\gamma_{D}^{\infty, N}$. This completes the proof.

\section{G. Proof of Corollary 9}

We use a similar approach as done in Appendix E. At first, we express the approximated conditional CDF expression of $F_{\gamma_{R}^{\text {,ins }} \mid L_{t r}}^{\infty}\left(\gamma_{\mathrm{th}}, L_{t r}\right)$ with respect to $L_{t r}$ as (cf. (35))

$$
\begin{aligned}
F_{\gamma_{R}^{\prime, \text { ins }} \mid L_{t r}}^{\infty}\left(\gamma_{\mathrm{th}}, L_{t r}\right)=\frac{1}{P} \\
\times \frac{(1-\alpha) \gamma_{\mathrm{th}} P_{0} L_{t r}}{2 \eta \alpha \chi_{s r}}\left(\sum_{i=1}^{N+1} \sum_{j=2}^{m_{i s}} \frac{\Phi_{s}^{\mathcal{N}}(i, j)}{(j-1) \chi_{i s}}\right. \\
\left.-\sum_{i=1}^{N+1} \frac{\Phi_{s}^{\mathcal{N}}(i, 1)}{\chi_{i s}} \ln \left(\frac{(1-\alpha) \gamma_{\mathrm{th}} P_{0} L_{t r}}{2 \eta \alpha \chi_{s r} \chi_{i s} P}\right)\right) .
\end{aligned}
$$

The unconditional CDF expression is then obtained as

$$
F_{\gamma_{R}^{N, \text { ins }}}^{\infty}\left(\gamma_{\text {th }}\right)=\int_{0}^{\infty} F_{\gamma_{R}^{\mathcal{N} \text {,ins }} \mid L_{t r}}^{\infty}\left(\gamma_{\text {th }}, x\right) f_{L_{t r}}(x) d x
$$

With the help of [31, Eq. (3.381.4)] and [31, Eq. (4.352.1)], we can calculate the resulting integrals in (G.2). The result in (43) is thus proved.

\section{H. Proof of Corollary 12}

Let us start by noting that the Taylor series expansion of $1 /(a+$ $x)$ at $x=0$ can be expressed as $1 /(a+x)=1 / a-x / a^{2}+o\left(x^{2}\right)$. Therefore, we can approximate the integral $\Theta(a, n, b)$ defined in Proposition 10 when $b \rightarrow 0$ as

$$
\begin{aligned}
\Theta(a, n, b) & \triangleq \int_{0}^{a} \frac{x^{n} e^{-x}}{x+b} d x \approx \int_{0}^{a} x^{n} e^{-x}\left(\frac{1}{x}-\frac{b}{x^{2}}\right) d x \\
& =\gamma(n, a)-b \gamma(n-1, a) .
\end{aligned}
$$

When $P \rightarrow \infty$ and $I=\lambda P$, the exact CDF expression in (44) can be approximated to

$$
\begin{aligned}
& F_{\gamma_{R}^{\text {Nave }}}\left(\gamma_{\mathrm{th}}\right) \approx 1-\sum_{i=1}^{N+1} \sum_{j=1}^{m_{i s}} \frac{\Phi_{s}^{\mathcal{N}}(i, j)}{(j-1) !} \\
& \times\left[\gamma\left(j, \frac{I \Xi}{\chi_{s p} \chi_{i s} \ln (1 / \epsilon)}\right)-\frac{\gamma_{\mathrm{th}} \Xi P_{0} \chi_{t r}}{\chi_{s p} \chi_{i s}}\right. \\
& \times \gamma\left(j-1, \frac{I \Xi}{\chi_{s p} \chi_{i s} \ln (1 / \epsilon)}\right) \\
& +\left(1-\frac{\gamma_{\mathrm{th}} P_{0} \chi_{s p} \chi_{t r} \ln (1 / \epsilon)}{\gamma_{\mathrm{th}} P_{0} \chi_{s p} \chi_{t r} \ln (1 / \epsilon)+I \chi_{s r}}\right) \\
& \left.\times \Gamma\left(j, \frac{I \Xi}{\chi_{s p} \chi_{i s} \ln (1 / \epsilon)}\right)\right]
\end{aligned}
$$

By using the equation of $\gamma(n, a)+\Gamma(n, a)=\Gamma(n)$, the property of $\sum_{i=1}^{N+1} \sum_{j=1}^{m_{i s}} \Phi_{s}^{\mathcal{N}}(i, j)=1$, and the Laurent series expansion of $a /(x+a)$ at $x=\infty$, that is, $a /(x+a)=a / x+o\left((1 / x)^{2}\right)$, we can simplify (H.2) to (46). This completes the proof. 


\section{Conflicts of Interest}

The author declares that there are no conflicts of interest regarding the publication of this paper.

\section{References}

[1] X. Lu, P. Wang, D. Niyato, D. I. Kim, and Z. Han, "Wireless networks with RF energy harvesting: a contemporary survey," IEEE Communications Surveys \& Tutorials, vol. 17, no. 2, pp. 757-789, 2015.

[2] S. Sudevalayam and P. Kulkarni, "Energy harvesting sensor nodes: survey and implications," IEEE Communications Surveys and Tutorials, vol. 13, no. 3, pp. 443-461, 2011.

[3] R. Zhang and C. K. Ho, "MIMO broadcasting for simultaneous wireless information and power transfer," IEEE Transactions on Wireless Communications, vol. 12, no. 5, pp. 1989-2001, 2013.

[4] K. Huang and V. K. N. Lau, "Enabling wireless power transfer in cellular networks: architecture, modeling and deployment," IEEE Transactions on Wireless Communications, vol. 13, no. 2, pp. 902-912, 2014.

[5] C. Zhong, X. Chen, Z. Zhang, and G. K. Karagiannidis, "Wireless-powered communications: performance analysis and optimization," IEEE Transactions on Communications, vol. 63, no. 12, pp. 5178-5190, 2015.

[6] Y. Ma, H. Chen, Z. Lin, Y. Li, and B. Vucetic, "Distributed and optimal resource allocation for power beacon-assisted wirelesspowered communications," IEEE Transactions on Communications, vol. 63, no. 10, pp. 3569-3583, 2015.

[7] J. Zhang, N.-P. Nguyen, J. Zhang, E. Garcia-Palacios, and N. P. Le, "Impact of primary networks on the performance of energy harvesting cognitive radio networks," IET Communications, vol. 10, no. 18, pp. 2559-2566, 2016.

[8] Y. Liu, L. Wang, S. A. R. Zaidi, M. Elkashlan, and T. Q. Duong, "Secure D2D communication in large-scale cognitive cellular networks: a wireless power transfer model," IEEE Transactions on Communications, vol. 64, no. 1, pp. 329-342, 2016.

[9] N. P. Le, N.-S. Vo, M.-T. Hoang, and D.-D. Tran, "Unified analysis of energy harvesting-based MIMO relay wireless systems over Nakagami-m fading channels," Transactions on Emerging Telecommunications Technologies, 2017.

[10] T. X. Doan, T. M. Hoang, T. Q. Duong, and H. Q. Ngo, "Energy harvesting-based D2D communications in the presence of interference and ambient RF sources," IEEE Access, vol. 5, pp. 5224-5234, 2017.

[11] A. A. Nasir, X. Zhou, S. Durrani, and R. A. Kennedy, "Relaying protocols for wireless energy harvesting and information processing," IEEE Transactions on Wireless Communications, vol.12, no. 7, pp. 3622-3636, 2013.

[12] Z. Hadzi-Velkov, N. Zlatanov, T. Q. Duong, and R. Schober, "Rate maximization of decode-and-forward relaying systems with RF energy harvesting," IEEE Communications Letters, vol. 19, no. 12, pp. 2290-2293, 2015.

[13] G. Zhu, C. Zhong, H. A. Suraweera, G. K. Karagiannidis, Z. Zhang, and T. A. Tsiftsis, "Wireless information and power transfer in relay systems with multiple antennas and interference," IEEE Transactions on Communications, vol. 63, no. 4, pp. 1400-1418, 2015.

[14] Z. Ding, I. Krikidis, B. Sharif, and H. V. Poor, "Wireless information and power transfer in cooperative networks with spatially random relays," IEEE Transactions on Wireless Communications, vol. 13, no. 8, pp. 4440-4453, 2014.
[15] D. S. Michalopoulos, H. A. Suraweera, and R. Schober, "Relay selection for simultaneous information transmission and wireless energy transfer: a tradeoff perspective," IEEE Journal on Selected Areas in Communications, vol. 33, no. 8, pp. 1578-1594, 2015.

[16] Y. Liu, L. Wang, M. Elkashlan, T. Q. Duong, and A. Nallanathan, "Two-way relay networks with wireless power transfer: design and performance analysis," IET Communications, vol. 10, no. 14, pp. 1810-1819, 2016.

[17] A. Goldsmith, S. A. Jafar, I. Maric, and S. Srinivasa, "Breaking spectrum gridlock with cognitive radios: an information theoretic perspective," Proceedings of the IEEE, vol. 97, no. 5, pp. 894-914, 2009.

[18] S. Lee, R. Zhang, and K. Huang, "Opportunistic wireless energy harvesting in cognitive radio networks," IEEE Transactions on Wireless Communications, vol. 12, no. 9, pp. 4788-4799, 2013.

[19] M. Zheng, C. Xu, W. Liang, and H. Yu, "Harvesting-throughput tradeoff for RF-powered underlay cognitive radio networks," Electronics Letters, vol. 52, no. 10, pp. 881-883, 2016.

[20] V. Rakovic, D. Denkovski, Z. Hadzi-Velkov, and L. Gavrilovska, "Optimal time sharing in underlay cognitive radio systems with RF energy harvesting," in Proceedings of the IEEE International Conference on Communications, ICC '15, pp. 7689-7694, London, UK, 2015.

[21] S. S. Kalamkar, J. P. Jeyaraj, A. Banerjee, and K. Rajawat, "Resource allocation and fairness in wireless powered cooperative cognitive radio networks," IEEE Transactions on Communications, vol. 64, no. 8, pp. 3246-3261, 2016.

[22] D. Xu and Q. Li, "Joint power control and time allocation for wireless powered underlay cognitive radio networks," IEEE Wireless Communications Letters, vol. 6, no. 3, pp. 294-297, 2017.

[23] T. Q. Duong, D. B. Da Costa, T. A. Tsiftsis, C. Zhong, and A. Nallanathan, "Outage and diversity of cognitive relaying systems under spectrum sharing environments in nakagami-m fading," IEEE Communications Letters, vol. 16, no. 12, pp. 20752078, 2012.

[24] Y. Liu, S. A. Mousavifar, Y. Deng, C. Leung, and M. Elkashlan, "Wireless energy harvesting in a cognitive relay network," IEEE Transactions on Wireless Communications, vol. 15, no. 4, pp. 2498-2508, 2016.

[25] Z. Yang, Z. Ding, P. Fan, and G. K. Karagiannidis, "Outage performance of cognitive relay networks with wireless information and power transfer," IEEE Transactions on Vehicular Technology, vol. 65, no. 5, pp. 3828-3833, 2016.

[26] Z. Wang, Z. Chen, B. Xia, L. Luo, and J. Zhou, "Cognitive relay networks with energy harvesting and information transfer: design, analysis, and optimization," IEEE Transactions on Wireless Communications, vol. 15, no. 4, pp. 2562-2576, 2016.

[27] C. Xu, M. Zheng, W. Liang, H. Yu, and Y.-C. Liang, "Outage performance of underlay multihop cognitive relay networks with energy harvesting," IEEE Communications Letters, vol. 20, no. 6, pp. 1148-1151, 2016.

[28] V.-D. Nguyen, S. Dinh-Van, and O.-S. Shin, “Opportunistic relaying with wireless energy harvesting in a cognitive radio system," in Proceedings of the IEEE Wireless Communications and Networking Conference, WCNC '15, pp. 87-92, New Orleans, LA, USA, 2015.

[29] G. K. Karagiannidis, N. C. Sagias, and T. A. Tsiftsis, "Closedform statistics for the sum of squared Nakagami-m variates and its applications," IEEE Transactions on Communications, vol. 54, no. 8, pp. 1353-1359, 2006. 
[30] P. J. Smith, P. A. Dmochowski, H. A. Suraweera, and M. Shafi, "The effects of limited channel knowledge on cognitive radio system capacity," IEEE Transactions on Vehicular Technology, vol. 62, no. 2, pp. 927-933, 2013.

[31] I. S. Gradshteyn and I. M. Ryzhik, Table of Integrals, Series and Products, Academic Press, Russia, 2007. 


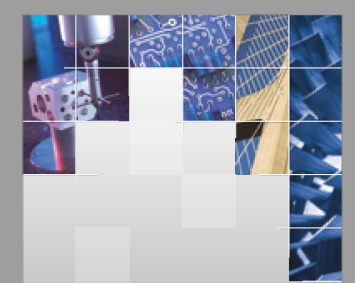

\section{Enfincering}
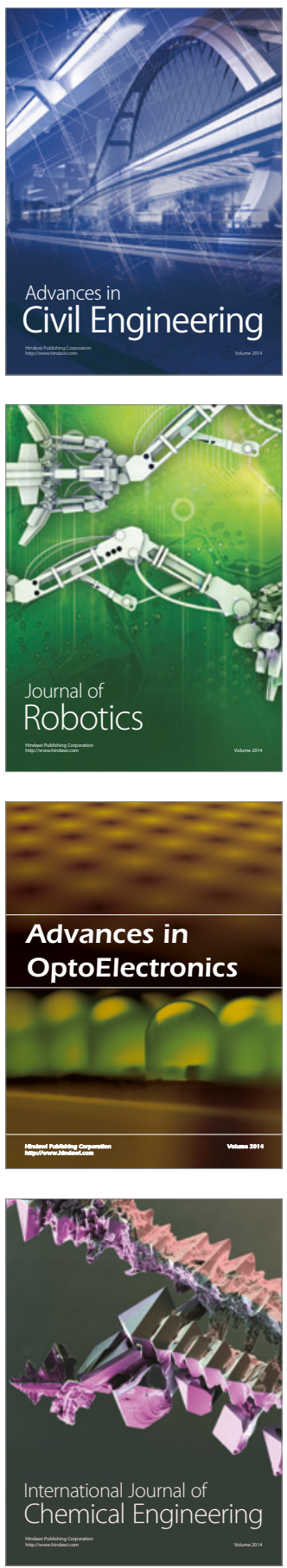

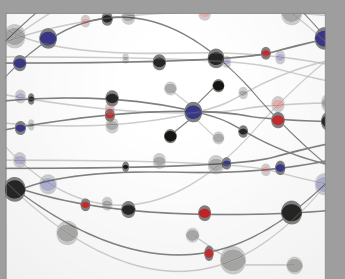

The Scientific World Journal

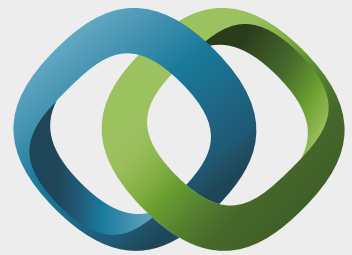

\section{Hindawi}

Submit your manuscripts at

https://www.hindawi.com
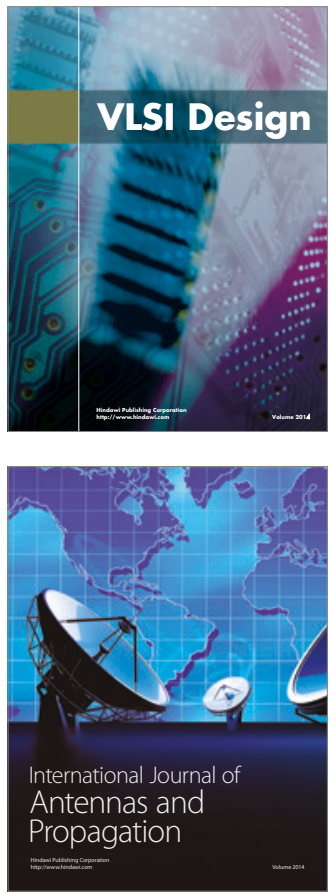

\section{Rotating}

Machinery
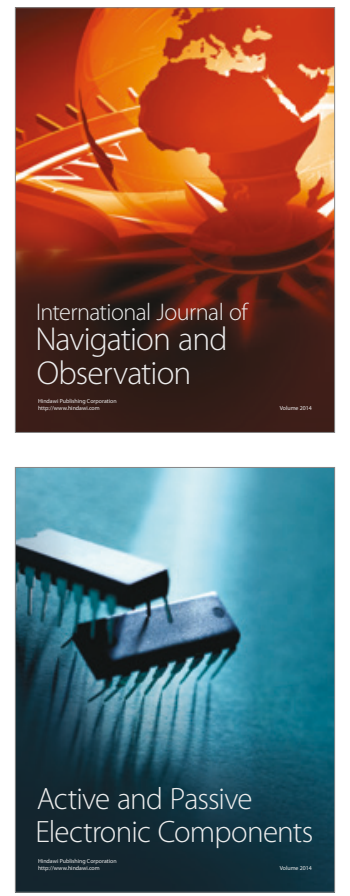
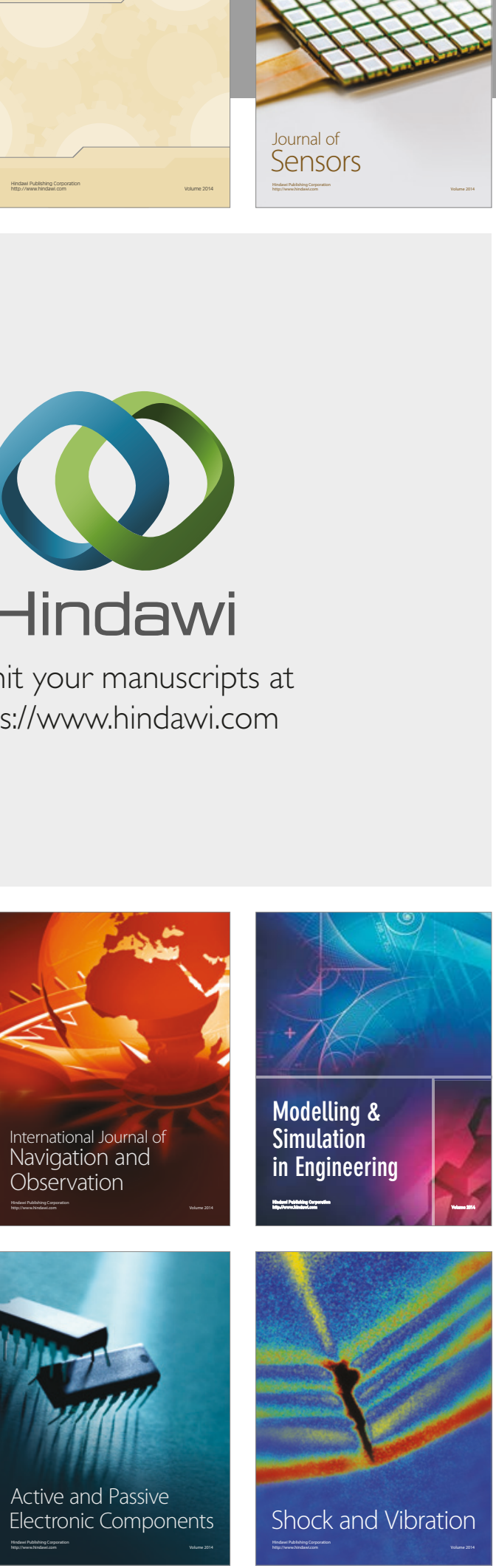
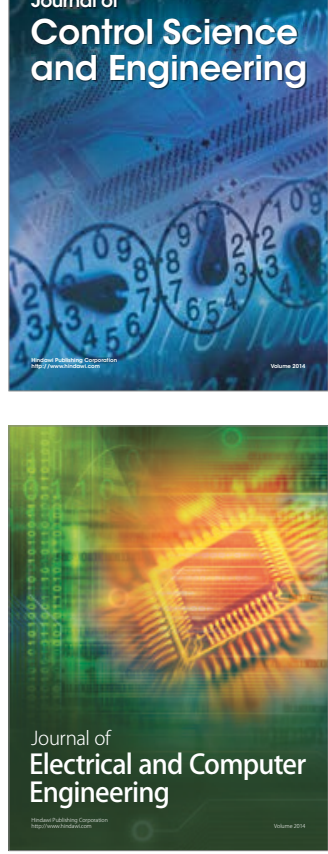

Distributed

Journal of

Control Science

and Engineering
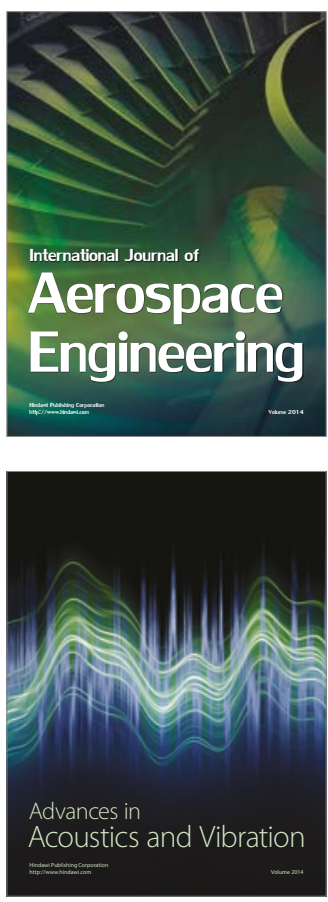

Sensor Networks 